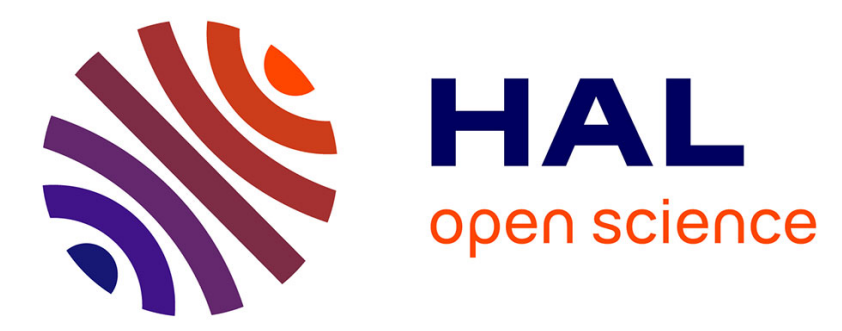

\title{
La datation archéomagnétique des matériaux de construction d'argile cuite: apports chronologiques et technologiques \\ Philippe Lanos
}

\section{- To cite this version:}

Philippe Lanos. La datation archéomagnétique des matériaux de construction d'argile cuite: apports chronologiques et technologiques. Gallia - Fouilles et monuments archéologiques en France métropolitaine, 1990, 47, pp.321-341. 10.3406/galia.1990.2914 . halshs-00396650

\section{HAL Id: halshs-00396650 \\ https://shs.hal.science/halshs-00396650}

Submitted on 20 Jan 2020

HAL is a multi-disciplinary open access archive for the deposit and dissemination of scientific research documents, whether they are published or not. The documents may come from teaching and research institutions in France or abroad, or from public or private research centers.
L'archive ouverte pluridisciplinaire HAL, est destinée au dépôt et à la diffusion de documents scientifiques de niveau recherche, publiés ou non, émanant des établissements d'enseignement et de recherche français ou étrangers, des laboratoires publics ou privés.

\section{(ㅇ)(1) $\$$}

Distributed under a Creative Commons Attribution - NonCommercial - NoDerivatives| 4.0 


\title{
LA DATATION ARCHÉOMAGNÉTIQUE DES MATÉRIAUX DE CONSTRUCTION D'ARGILE CUITE Apports chronologiques et technologiques
}

\author{
par Philippe LANOS
}

\begin{abstract}
Depuis peu, la datation archéomagnétique s'applique aux matériaux de construction d'argile cuite tels que les luiles, les briques et les carreaux de pavage. L'exposé des principes physiques et statistiques de l'archéomagnétisme et la présentation complète d'une étude expérimentale portant sur des carreaux de pavage modernes montre la grande fiabilité et précision de la méthode. Ainsi, on peut obtenir, en France, des dates avec une précision de \pm 20 ans pour la majeure partie des périodes allant de la fin de la protohistoire à nos jours. Des exemples d'application viennent illustrer les nombreux apports chronologiques de la méthode. Enfin, on montre comment l'archéomagnétisme permet d'obtenir des renseignements archéologiques et technologiques à partir de l'étude des positions de cuisson des matériaux et des caractéristiques magnétiques des argiles.
\end{abstract}

The archaeomagnetic dating is now applied to building materials of baked clay such as tiles, bricks and paving tiles. The presentation of the physical and statistical principles and the complete presentation of an experimental study concerning modern paving tiles show the great reliability and precision of the method. Thus, we can obtain, in France, dates with a precision of \pm 20 years for the major part of the periods from the end of the protohistory till now. Application examples illustrate the multiple chronological contributions of the method. Finally, we show how archaeomagnetism allows to obtain archaeological and technological informations from study of the firing positions of the materials and the magnetic characteristics of the clays.

La datation archéomagnétique repose d'une part sur l'existence dans le passé d'un Champ Magnétique Terrestre (CMT) variable dans le temps et d'autre part sur la propriété qu'ont les argiles d'acquérir, au cours du refroidissement consécutif à tout chauffage, une aimantation parallèle et proportionnelle à ce CMT. La condition du parallélisme entre l'aimantation induite et le champ ambiant constitue le fondement de la méthode. L'archéomagnétisme est né en France, dès l'avant-guerre, grâce aux travaux précurseurs d'E. Thellier $(1938,1971$ et 1981). Les récents progrès concernant la reconstitution de la variation directionnelle du CMT dans le passé rendent la méthode de datation par l'archéomagnétisme apte à fournir, en France, des dates avec une précision de \pm 20 ans pour la majeure partie des périodes allant de la fin de la protohistoire à nos jours.

La méthode s'applique en premier lieu aux vestiges de fours, de foyers et de constructions incendiées ou fortement chauffées qui ont conservé la position de la dernière chauffe. Le prélèvement sur ces structures dites "en place» d'échantillons orientés in situ permet, après mesure de l'aimantation au laboratoire, de déterminer la direction du CMT ayant régné au lieu et à la date de la dernière cuisson (Thellier, 1981). Depuis peu, la méthode s'applique aussi aux matériaux dits "déplacés», dont le Laboratoire d'Archéométrie fait sa spécialité. Il s'agit de matériaux d'argile cuite déplacés du lieu de leur fabrication (l'atelier) au lieu de leur utilisation (la construction). Les applications actuelles concernent principalement les poteries et les matériaux de construction tels que les tuiles, les briques, les carreaux de pavage, ou encore les tubuli et les éléments de briquetages. De tels matériaux ne sont utilisables à des fins de datation par l'archéomagnétisme que s'il est possible de retrouver, à partir de considérations technologiques simples et de bon sens, la position qu'ils avaient lors de leur dernière cuisson. 


\section{PRINCIPES DE LA MÉTHODE}

La Variation SÉCUlaire dU CHAMP MaGNÉtiQUE TERRESTRE

Le globe terrestre possède un champ magnétique que l'on peut matérialiser en tout point entourant la terre par son action sur l'aiguille aimantée d'une boussole libre de s'orienter autour de son centre de gravité. Ainsi, en un point 0 de la surface de la terre, à l'instant $t$. le CMT peut être représenté par un vecteur $\overrightarrow{O F}$ dont la direction est donnée par celle de l'aiguille aimantée. Dans un repère géographique local (Nord, Est, Verticale du lieu), cette direction est complètement définie par deux angles : la déclinaison $\mathrm{D}$ et l'inclinaison I (fig. 1). La variation séculaire de la direction a fait l'objet de mesures directes dès les annécs 1600 à Londres, Paris et Rome (Bauer, 1899). L'examen des données des trois derniers siècles (Aitken, 1974; Thellier, 1981; Hoye, 1981; Tanguy, Bucur, Thompson, 1985; Bucur, 1986) montre une assez grande homogénéité de la variation séculaire de l'inclinaison dans un domaine allant de l'Italie aux Iles Britanniques. Les courbes présentent un bon parallélisme, l'écart provenant essentiellement de la différence de latitude entre les sites. En effet, il est important de noter qu'à un même instant, l'inclinaison varie en fonction de la latitude du lieu de mesure. Il est possible cependant de comparer des mesures effectuées en des lieux différents grâce à une correction de latitude basée sur un modèle physique simple du CMT. Cette observation justifie la réduction en un lieu commun (Paris pour la France) des mesures obtenues à partir de sites répartis sur l'aire géographique précitée.

La reconstitution de la variation séculaire de l'inclinaison et de la déclinaison, avant le xvire $\mathrm{s}$., repose sur la mesure de la direction de l'aimantation mémorisée par des argiles cuites bien datées par le contexte archéologique ou les documents historiques. Les premières mesures des éléments du CMT sur des argiles cuiles bien datées par les archéologues furent entreprises par le Professeur E. Thellier dès l'avantguerre (1938). La synthèse d'une quarantaine d'années de travail (Thellier, 1981) a permis.à ce dernier de proposer une reconstitution de la variation de la direction, en France, pour des périodes allant du début de notre ère au XVII ${ }^{\mathrm{e}}$ s. (fig. 2), avec cependant une importante lacune entre le $v^{\mathrm{e}}$ et le viII $\mathrm{s}$.

Les laboratoires de Géomagnétisme de SaintMaur-des-Fossés et d'Archéométrie de Rennes ont récemment apporté des données complémentaires permettant d'affiner la courbe de Thellier pour les époques gallo-romaine (Langouët, Bucur, Goulpeau,

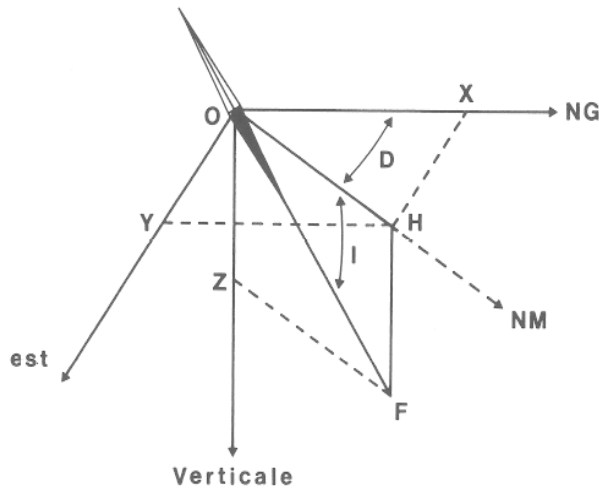

Fig. 1 - Inclinaison I par rapport au plan horizontal local (Oxy), Déclinaison D par rapport au Nord géographique (NG) et Intensité $\mathrm{F}$ du Champ Magnétique Terrestre (CMT) dans le repère géographique local Nord, Est, Verticale du lieu. A titre d'exemple, le CMT à Paris, en 1975, était caractérisé par une inclinaison de $64^{\circ}$ (vers le bas), une déclinaison de $5^{\circ} \mathrm{W}$ et une intensité de $47000 \gamma$.

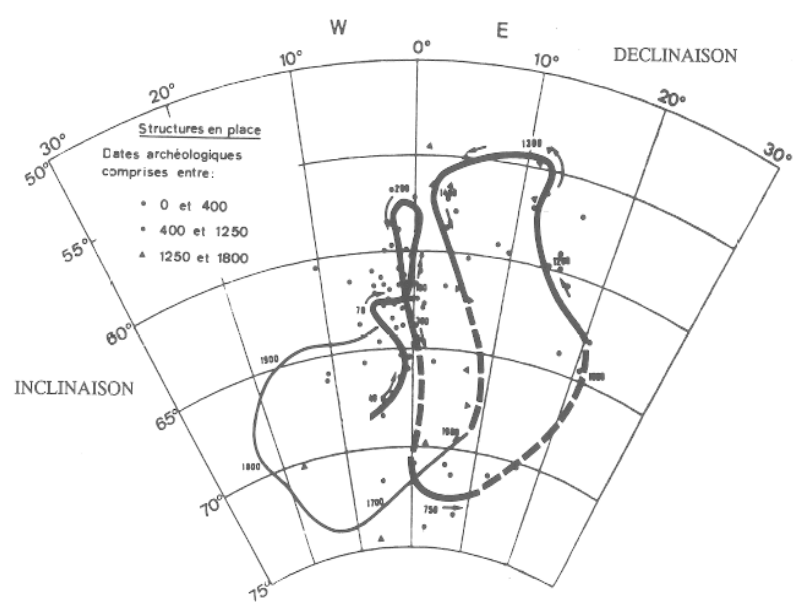

Fig. 2 - Variation séculaire de la direction du CMT, en France, durant la période historique (d'après Thellier, 1981).

1983; Lanos, Goulpeau, Bucur, Langouët, 1990) et médiévale (Bucur, 1986). Durant toute la période historique, l'inclinaison a fluctué entre $55^{\circ}$ et $75^{\circ}$ et la déclinaison entre $23^{\circ} \mathrm{W}$ et $20^{\circ} \mathrm{E}$. La courbe de variation séculaire de l'inclinaison du CMT durant l'époque gallo-romaine (fig. 3) montre l'existence d'une double oscillation avec deux maxima atteints un peu avant notre ère et au milieu du III $^{\mathrm{e}} \mathrm{s}$. et deux minima atteints à la fin du II $^{\mathrm{e}} \mathrm{s}$. et au milieu du Iv $^{\mathrm{e}} \mathrm{s}$. La précision des datations que l'on peut déduire d'une telle courbe est de l'ordre de \pm 20 ans en moyenne. Mais en contrepartie de cette excellente précision, plusieurs dates peuvent être proposées 


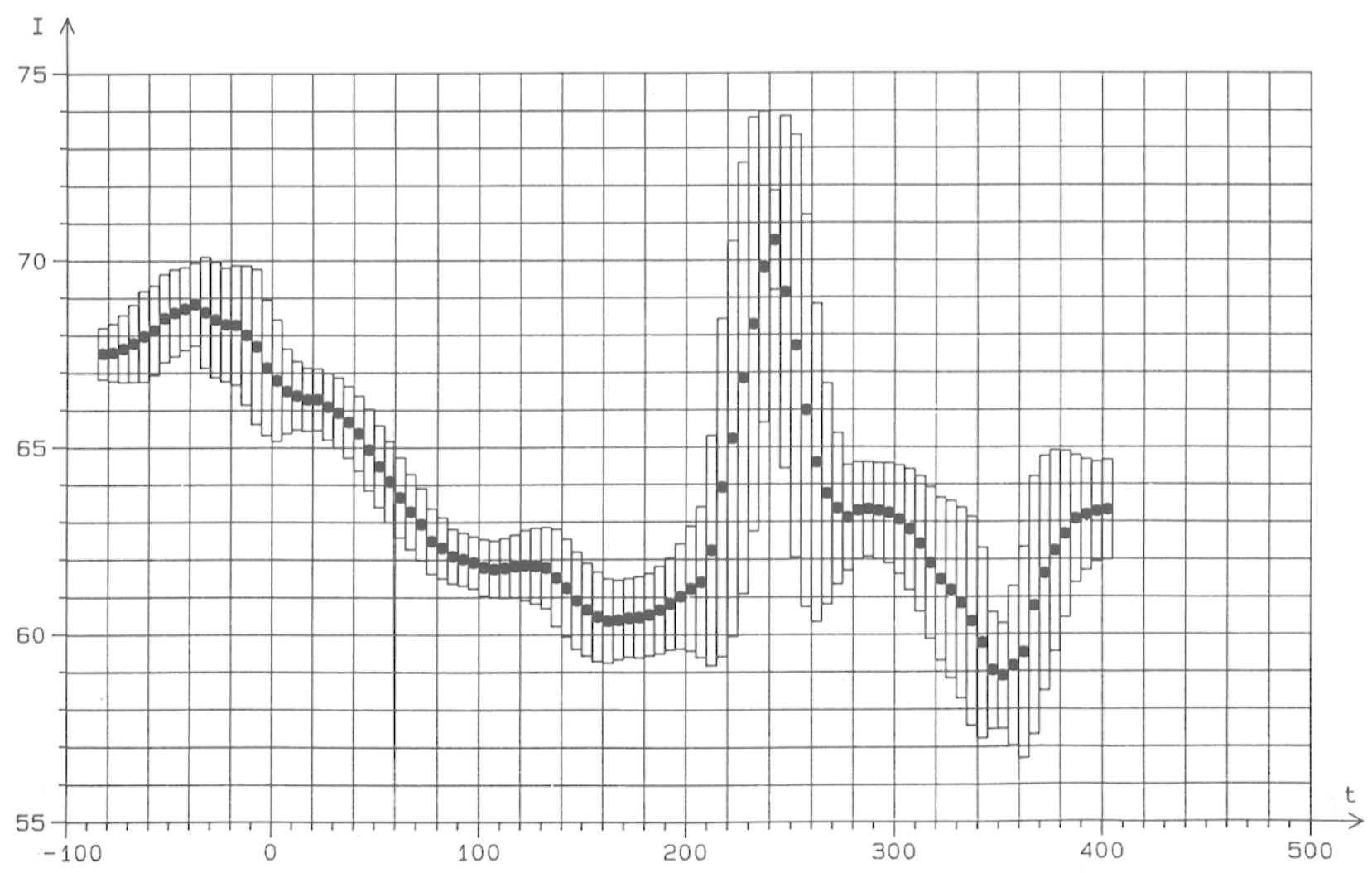

Fig. 3 - Variation séculaire de l'inclinaison du CMT, en France, durant l'époque romaine (d'après Lanos, Goulpeau, Bucur et Langouët, à paraître).

pour une même valeur de l'inclinaison. Selon la valeur mesurée et selon les données archéologiques disponibles, le choix entre les différentes solutions peut être plus ou moins facile, comme on pourra le voir dans les différents exemples donnés plus loin.

\section{L'Aimantation THERMORÉmANENTE DES ARGILES CUITES}

Le processus physique à l'origine de l'aimantation des argiles cuites (on parle d'aimantation rémanente) consiste en un chauffage à une température donnée, suivi d'un refroidissement en présence d'un champ magnétique extérieur. L'argile cuite acquiert alors une aimantation, dite thermorémanente (ATR), de même direction et sens que le champ ambiant et d'intensité proportionnelle à celui-ci. L'unité de mesure de l'intensité d'aimantation est l'"Ampère par mètre", noté $\mathrm{A} / \mathrm{m}$. Les lois générales de l'aimantation des argiles cuites furent découvertes par $\mathrm{E}$. Thellier (1938) et ce fut L. Néel (1949) qui le premier en établit une théorie physique quantitative.

Les minéraux magnétiques (hématite, magnétite ...) sont présents dans les argiles essentiellement sous la forme de grains extrêmement fins (quelques microns), dans une proportion de quelques pour cent. Chaque grain possède une aimantation individuelle, que l'on peut comparer à l'aiguille aimantée d'une boussole, stable tant que la température ambiante ne dépasse pas une certaine température de blocage caractéristique du grain. L'aimantation observée à l'échelle de l'échantillon (aimantation macroscopique) est la somme des aimantations individuelles portées par des milliers de grains fins dispersés au hasard dans la matrice argileuse. Lorsque l'argile est chauffée à une température supérieure à la température dite de Curie $\mathrm{T}_{c}$ (inférieure ou égale à $680^{\circ} \mathrm{C}$ selon la nature des minéraux magnétiques mis en jeu), les petites aimantations de tous les grains s'annulent. Il se produit un effacement total de toute ATR acquise antérieurement. Dès que la température redescend au-dessous de $T_{c}$, les grains reprennent leurs aimantations individuelles. Si le refroidissement a lieu en l'absence de champ magnétique extérieur (champ nul), alors chaque grain reprendra une aimantation individuelle qui restera bloquée selon une direction propre. Les grains étant dispersés aléatoirement dans la matrice argileuse, la somme de leurs aimantations sera nulle. Il n'y aura pas d'ATR 
observée à l'échelle macroscopique. Par contre, si le refroidissement a lieu en présence d'un champ extérieur, le CMT par exemple, les aimantations individuelles d'une partie des grains se bloqueront dans la direction du champ appliqué et garderont cette orientation de façon extrêmement stable au cours du temps (durant des millions d'années).

D'autre part, il est physiquement possible que plusieurs aimantations thermorémanentes, de directions différentes, coexistent au sein d'une même argile cuite. Le processus d'acquisition de ces ATR dites partielles et les applications archéologiques très importantes qui en découlent font l'objet d'un exposé détaillé en annexe.

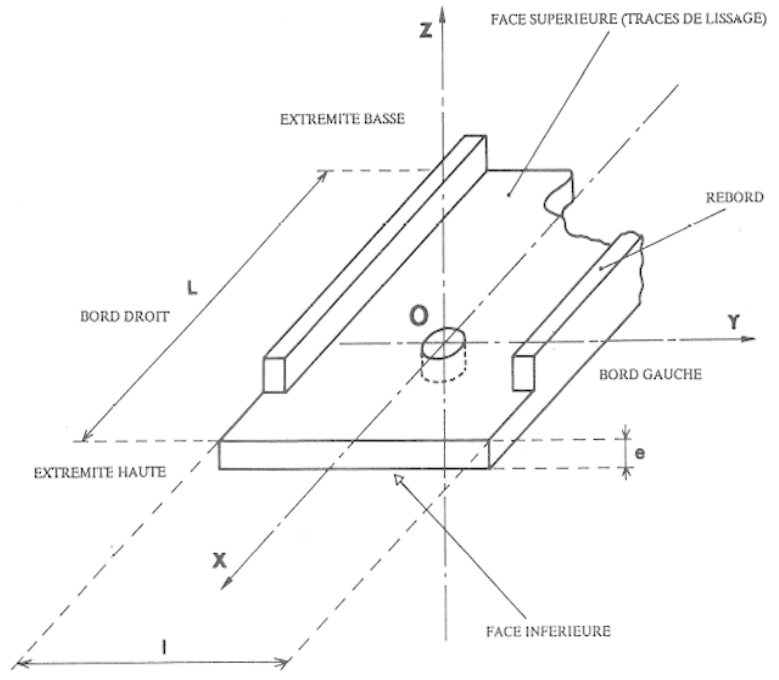

Fig. 4 - Repère de carottage $(0 x y z)$ associé à un fragment de tuile.

constructions sont des matériaux déplacés qui ont perdu la mémoire de leur position de cuisson dans le four. A ce stade, toute mesure de la direction de leur ATR serait inutile puisque non rattachable au repère géographique servant à définir la direction du C.MT. En fait, pour des raisons de commodité et de logique de remplissage, il apparaît raisonnable de supposer que ces matériaux de forme parallélépipédique reposaient sur l'une de leurs faces planes lors de la cuisson. Cette hypothèse entraîne l'existence de trois positions théoriques possibles : à plat, de chant ou debout. Ainsi, dans la mesure où la sole du four était statistiquement horizontale et plane, la mémoire du plan horizontal est conservée par l'une des faces des matériaux. Cette dernière hypothèse sur l'horizontalité des soles est en fait presque toujours vérifiée dans le cas des fours gallo-romains (Le $\mathrm{Ny}, 1988$ ).

Dans la pratique, la mesure de l'aimantation sur un objet de forme parallélépipédique tel qu'une tuile ou une brique se fait à partir d'une carotte de $25 \mathrm{~mm}$ de diamètre prélevée dans un repère $(0 x y z)$ lié à l'objet comme indiqué sur la figure 4. Cette mesure permet de calculer la valeur de l'inclinaison pour chacune des positions théoriques simples envisageables, à savoir à plat, de chant et debout. Il s'avère qu'à nos latitudes, une seule de ces positions satisfait la condition d'une inclinaison comprise dans l'intervalle $\left(50^{\circ}, 75^{\circ}\right)$ de variation du CMT (fig. 2). En conséquence, ce test fournit la bonne valeur de l'inclinaison, qui sert à la datation, et restitue un renseignement technologique perdu : la position de cuisson.

Les tuiles et les briques retrouvées dans les 
INCLINAISON MOYENNE

NOTION DE DÉVIATION

Les résultats des mesures de direction d'aimantation effectuées sur les échantillons d'un lot cohérent sont rassemblés dans un diagramme dit de pointage (fig. 6), où l'inclinaison I varie radialement de $40^{\circ}$ à $90^{\circ}$ et où la déviation $\Delta$ représente l'angle entre l'une des faces verticales des matériaux et la direction du NM de l'époque de cuisson (Goulpeau, Langouët, 1980 et 1982; Lanos, 1987a). Un symbole plein indique que la face supérieure de l'objet (face lissée par convention, côté rebord dans le cas des tegulae) est tournée vers le Nord magnétique (NM). A l'inverse, un symbole vide indique que la face supérieure est tournée vers le Sud magnétique. Dans le cas de tuiles cuites debout par exemple (fig. 5), la déviation donne l'orientation relative par rapport au NM du plan vertical d'aplatissement du matériau. Par contre, puisqu'il s'agit de matériaux déplacés, l'angle entre le Nord magnétique et le Nord géographique, c'est-à-dire la déclinaison, reste inaccessible.

L'examen des diagrammes de pointage (fig. 6 , $11 \mathrm{c}, 12 \mathrm{a}, 13 \mathrm{a}, 14$ et 16c) montre lexistence de nuages de points plus ou moins concentrés autour de directions moyennes qu'il s'agit de déterminer. Cette dispersion des directions s'explique principalement par l'hétérogénéité de l'aimantation dans les argiles cuites, les erreurs commises lors du repérage et des mesures, la forme plus ou moins parallélépipédique des matériaux et la dispersion des positions réelles de cuisson autour des positions théoriques supposées. Des études archéomagnétiques menées sur des échantillonnages importants (la centaine d'éléments est un chiffre moyen) permettent de réduire l'influence de ces causes d'erreurs. En effet, l'analyse statistique montre que la précision de la détermination des directions moyennes est d'autant plus grande que le nombre des mesures est grand.

Grâce à des traitements statistiques appropriés (Lanos, 1987a), chacun des agglomérats de points observés dans le diagramme de pointage peut être caractérisé par une inclinaison moyenne $\overline{\mathrm{I}}$ et une déviation moyenne $\bar{\Delta}$. L'inclinaison moyenne obtenue permet de remonter, après correctionn de latitude, à la datation via l'utilisation des courbes archéomagnétiques de référence (fig. 3). Le regroupement des déviations autour de certaines valeurs moyennes révèle quant à lui un rangement extrêmement régulier des tuiles dans les laboratoires. En particulier, les nombreuses études de lots de tuiles gallo-romaines effectuées ont presque toujours montré la présence de deux groupements distincts dont les déviations moyennes sont quasi complémentaires

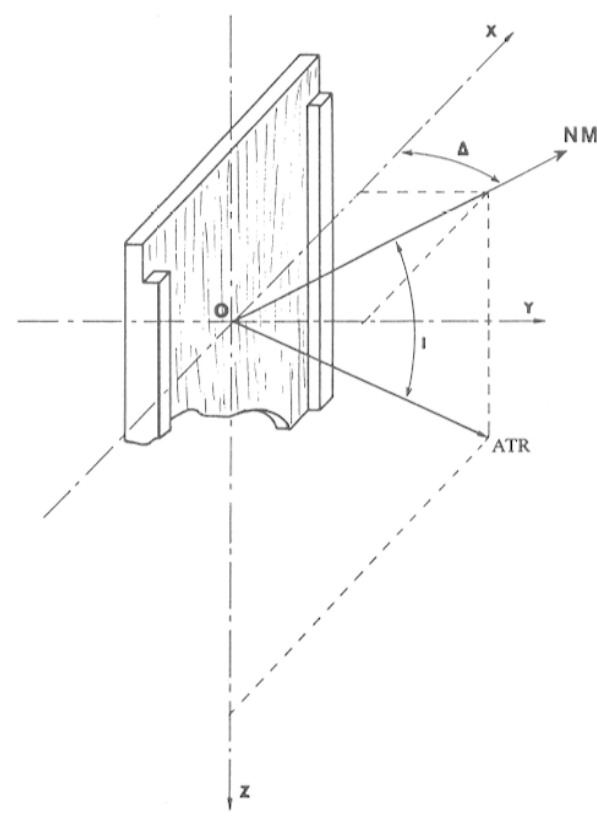

Fig. 5 - Inclinaison I et déviation $\Delta$ de l'aimantation mesurée sur une tuile cuite debout.

$\left(\mathrm{I} \bar{\Delta}-\bar{\Delta}^{\prime} \mathrm{I}=90^{\circ}\right)$. Si l'on suppose que les matériaux étaient rangés dans les fours parallèlement aux parois internes des laboratoires, alors les deux groupements peuvent être interprétés comme la conséquence d'une cuisson dans un four de forme rectangulaire dont les parois étaient orientées à $\bar{\Delta}$ et $\bar{\Delta}^{\prime}$ par rapport au NM de l'époque (fig. 7). Or, les observations archéologiques recueillies pour la Grande-Bretagne par A. Mc Whirr (1979) et pour la France par F. Le Ny (1988) montrent que les fours gallo-romains étaient majoritairement de forme rectangulaire. Ainsi, la concordance entre la forme des fours et l'existence d'agglomérats complémentaires dans les diagrammes archéomagnétiques résulte d'un rangement somme toute logique de matériaux de forme parallélépipédique parallèlement aux parois internes de fours de même forme.

A côté de lots de matériaux présentant un seul ensemble d'éléments fabriqués à une date unique et provenant d'un seul four caractérisé par les déviations moyennes $\bar{\Delta}$ et $\bar{\Delta}^{\prime}$, il existe des lots constitués d'un mélange de plusieurs ensembles différents généralement indiscernables à première vue. Ces lots sont recueillis sur des sites à histoire complexe : approvisionnements multiples à une même date, agrandissements, réparations ou récupérations. L'utilisation des diagrammes Inclinaison-Déviation permet en principe une séparation statistique de ces 


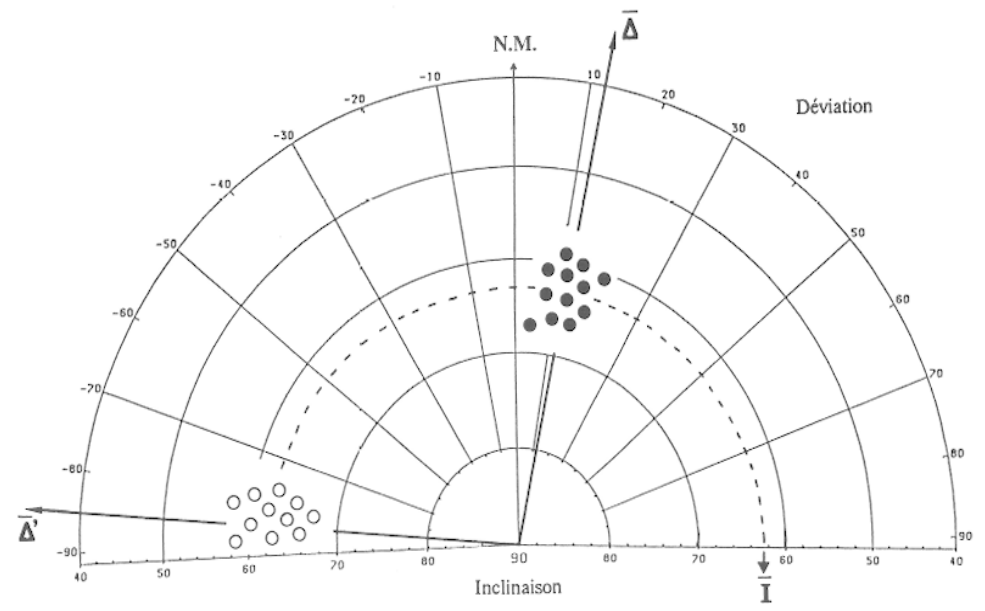

Fig. 6 - Diagramme de pointage des directions d'aimantation mesurées sur un lot de matériaux déplacés. Cet exemple théorique est caractérisé par deux agglomérats de déviations moyennes $\bar{\Delta}$ et $\bar{\Delta}^{\prime}$ complémentaires. La datation est obtenue à partir de l'inclinaison moyenne $\bar{I}$, en principe identique pour les deux agglomérats.

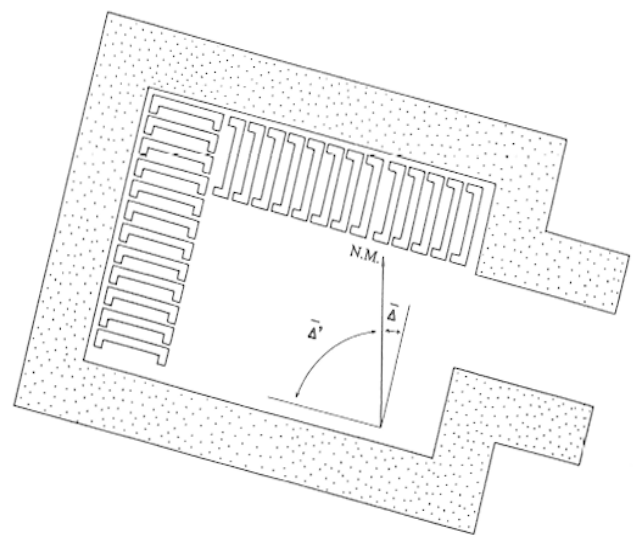

Fig. 7 - Schéma d'interprétation des données fournies par le diagramme de pointage de la figure 6 . ensembles, chacun étant caractérisé par une inclinaison moyenne permettant la datation, et deux déviations moyennes complémentaires témoins de la rectangularité des fours.

\section{EFFET DE FORME ET ANISOTROPIE D'AIMANTATION}

Il faut signaler l'existence de deux phénomènes magnétiques importants dont les effets perturbateurs peuvent détruire le parallélisme entre l'ATR induite et le CMT ambiant (fig. 8). Il s'agit d'une part du "champ démagnétisant", appelé encore "effet de forme", dont les effets apparaissent lorsque les matériaux sont fortement aimantés (aimantations supérieures à $5 \mathrm{~A} / \mathrm{m}$ ) et aplatis (rapports longueur/ épaisseur supérieurs à 5), et d'autre part de l'«anisotropie d'ATR», dont les effets interviennent lorsque les matériaux n'ont pas la même capacité à s'aimanter selon toutes les directions de l'espace.

L'anisotropie d'ATR d'un échantillon d'argile cuite de forme parallélépipédique est complètement caractérisée par les deux facteurs d'anisotropie $F_{x z}$ et $F_{x y}$. Ces facteurs sont déterminés expérimentalement au laboratoire par des opérations de chauffe et de refroidissement sous champ magnétique contrôlé. $\mathrm{F}_{\mathrm{xz}}$ est défini, pour un échantillon donné, comme le rapport entre l'aimantation acquise pour un champ appliqué selon l'axe $\mathrm{x}$ et l'aimantation acquise pour un champ de même intensité appliqué selon l'axe $z$ (fig. 4). $F_{x y}$ est défini de la même manière, l'axe $z$ étant remplacé par l'axe $y$. Les déterminations effectuées sur les échantillons de lots homogènes de

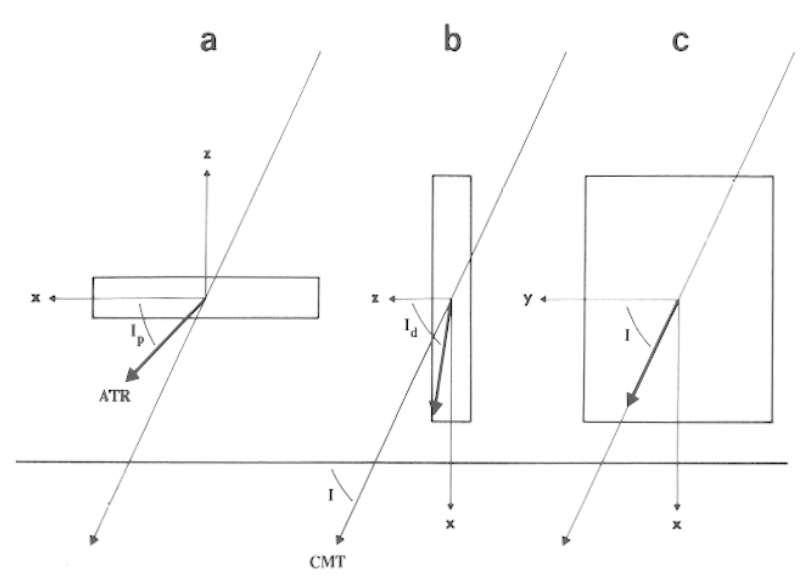

Fig. 8 - Effets du champ démagnétisant et de l'anisotropie d'aimantation thermorémanente sur la direclion de l'aimantation acquise par un objet fortement aimanté (aimantation supérieure à $5 \mathrm{~A} / \mathrm{m}$ ) et aplati (rapport longueur/èpaisseur supérieur à 5), peu allongé (rapport longueur/largeur voisin de 1), fortement anisotrope selon son épaisseur (facteur $\vec{F}_{x z}$ supérieur à 1), mais isotrope dans le plan d'aplatissement $\left(F_{x y}\right.$ voisin de 1$)$.

a) Position à plat : l'inclinaison $I_{p}$ de l'aimantation sera plus faible que l'inclinaison I du champ ambiant.

b) Position sur tranche (debout ou de chant) perpendiculairement au méridien magnétique (déviation proche de $90^{\circ}$ ) : l'inclinaison $I_{d}$ sera plus forte que $I$.

c) Position sur tranche parallèlement au méridien magnétique (déviation proche de $0^{\circ}$ ) : l'inclinaison sera quasiment identique à celle du CMT. En effet, dans cette situation, le rapport de forme de méme que le facteur $F_{x y}$ sont voisins de 1 . 
matériaux de construction montrent en général une très faible dispersion des valeurs de chacun des deux facteurs. On définit alors pour ces lots les facteurs d'anisotropie moyens $\bar{F}_{x z}$ et $\bar{F}_{x y}$. A titre d'exemple, $\overline{\mathrm{F}}_{\mathrm{xz}}=1,20$ signifie que les matériaux du lot considéré ont une capacité à s'aimanter selon l'axe $x$ en moyenne 1,2 fois plus forte que celle à s'aimanter selon l'axe $z$.

Grâce aux récents progrès de la méthode, l'application de formules théoriques dans le cas du champ démagnétisant (Lanos, 1987b) et la détermination expérimentale des facteurs d'anisotropie moyens $\overline{\mathrm{F}}_{\mathrm{xz}}$ et $\overline{\mathrm{F}}_{\mathrm{xy}}$ (Lanos, 1987a; Goulpeau, Lanos, Langouët, 1989) permettent de corriger ces éventuels effets et de retrouver la direction vraie du CMT ayant régné lors de la cuisson.

\section{VERIFICATION EXPERIMENTALE DE LA METHODE}

Cette étude expérimentale a porté sur un lot de carreaux de pavage fabriqués à la briqueterie Cailleau, aux Rairies (Maine-et-Loire), en janvier 1986. Cette briqueterie comprend quatre fours à tirage oblique avec sol incliné, fonctionnant de façon artisanale au feu de bois. Une vue en coupe du four ayant servi à cuire les carreaux étudiés ici est donnée (fig. 9).

Dans un premier temps, des observations directes ont été effectuées sur le terrain :

- la valeur de l'inclinaison du CMT, mesurée à l'extérieur et à l'intérieur du four avec l'aide d'un inclinomètre, était comprise entre 62 et $63^{\circ}$;

- la déviation de l'axe du four par rapport au $\mathrm{NM}$ était d'environ $20^{\circ} \mathrm{E}$; Nord :

- la pente du fond du four était de $8^{\circ}$ vers le

- les carreaux ont été fabriqués mécaniquement à l'aide d'une extrudeuse. L'extrusion de la pâte à travers une fente a provoqué un feuilletage et un étirement important de la pâte argileuse. L'étirement est matérialisé en surface par les stries de lissage, très visibles. Les dimensions des carreaux étudiés ici sont de $14 \times 14 \times 2 \mathrm{~cm}$ en moyenne;

- le chargement du four était constitué par une alternance de couches de carreaux de chant avec des couches de carreaux à plat (fig. 9). Le plan d'aplatissement des carreaux de chant était orienté parallèlement à la direction de pente, c'est-à-dire parallèlement à l'axe du four. Les carreaux à plat étaient rangés de telle sorte que les bords soient parallèles (respectivement perpendiculaires) à cet axe. On peut

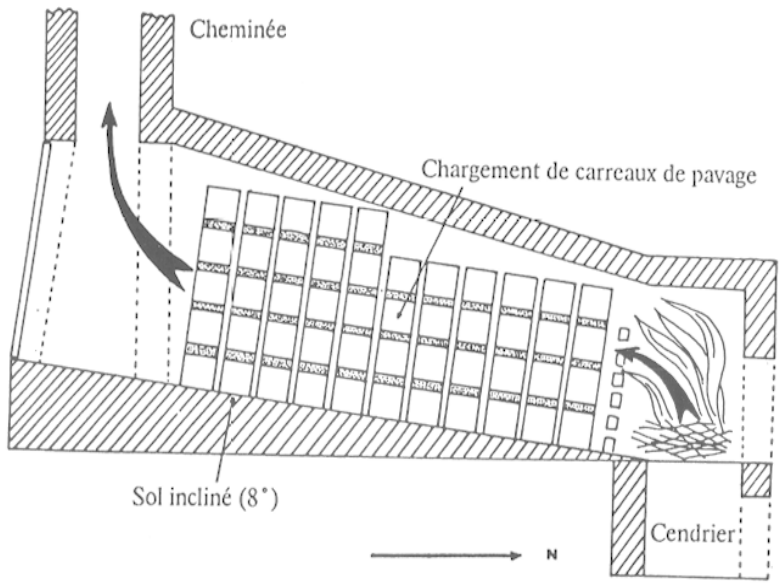

Fig. 9 - Vue en coupe d'un four à tirage oblique avec son chargement de carreaux de pavage (briqueterie Cailleau, Les Rairies, Maine-et-Loire).

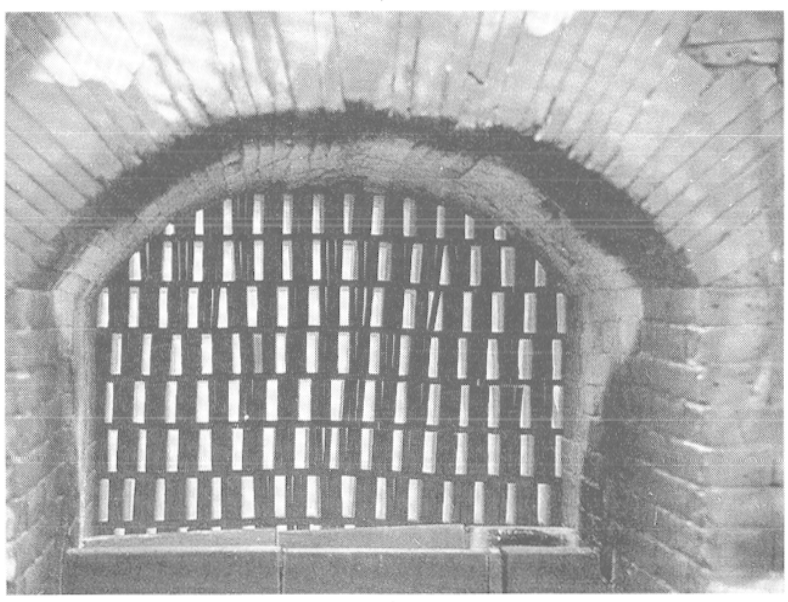

Fig. 10 - Cliché montrant un chargement de carreaux de pavage en cours de cuisson à une température supérieure à $1000{ }^{\circ} \mathrm{C}$ (briqueterie Cailleau, Les Rairies, Maine-et-Loire).

voir un chargement de ce type en cours de cuisson (fig. 10). La température régnant dans le four est supérieure à $1000^{\circ} \mathrm{C}$. Selon cette figure, $25 \%$ environ des carreaux sont à plat et $75 \%$ sont sur tranche.

L'étude archéomagnétique de 76 carreaux prélevés dans la partie centrale du four, lors du déchargement, a été effectuée au Laboratoire indépendamment des observations faites sur le terrain, sauf en ce qui concerne la pente. Les résultats des mesures montrent que $28 \%$ des carreaux ont été cuits à plat et que $72 \%$ l'ont été sur tranche. L'examen de l'histogramme des inclinaisons (fig. 11a) révèle une différence de près de $9^{\circ}$ entre l'inclinaison des matériaux cuits sur tranche et celle des matériaux cuits à plat. L'origine de cette différence ne peut 
a

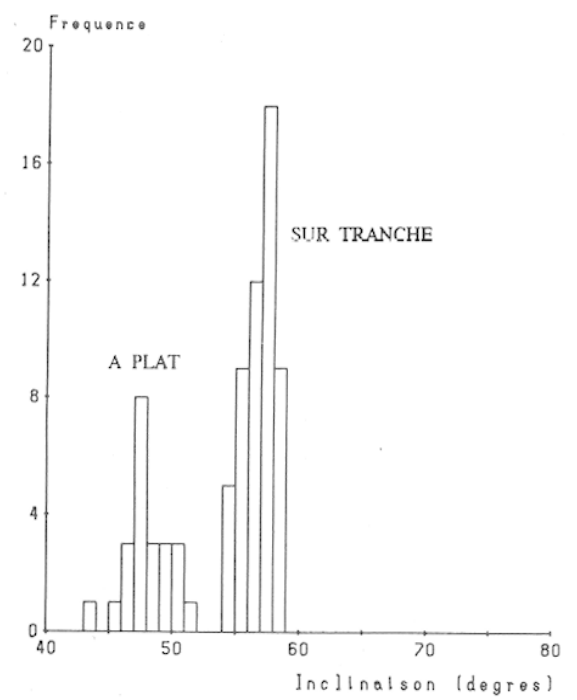

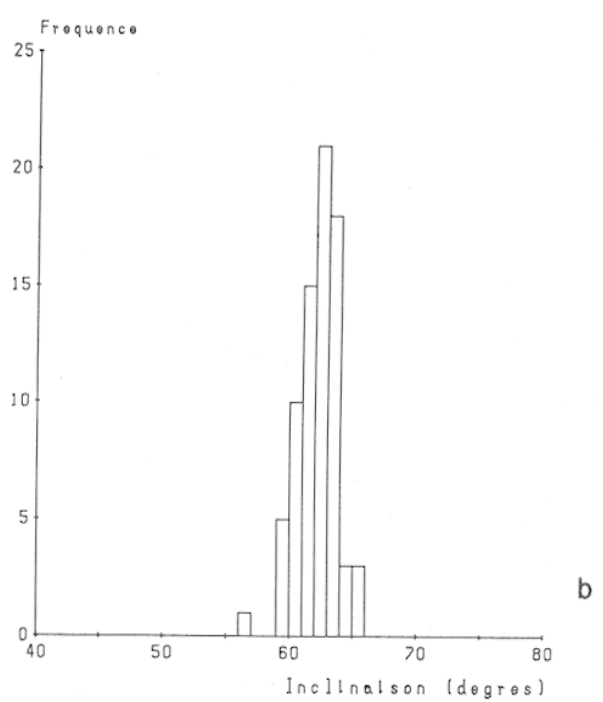

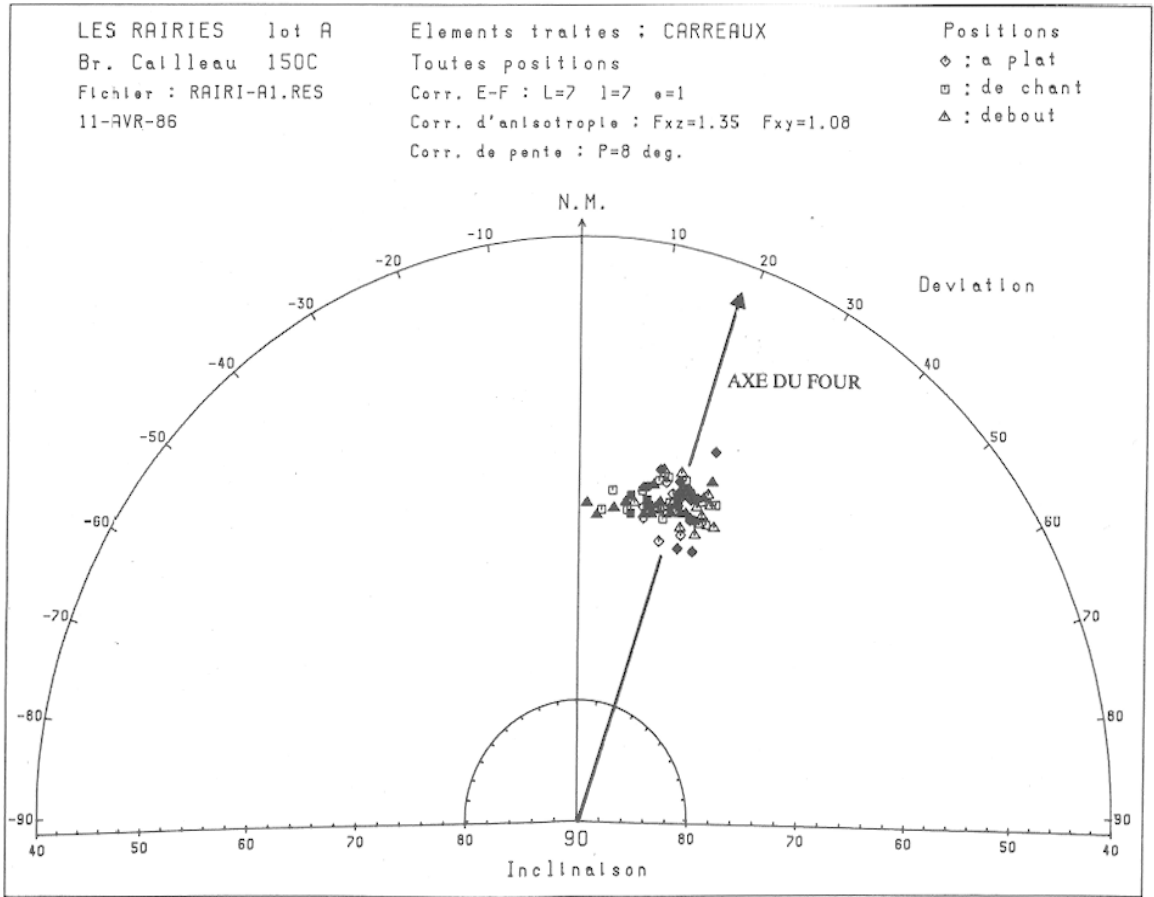

Fig. 11 - Vérification expérimentale de la méthode sur un lot de carreaux de pavage provenant de la briqueterie artisanale Cailleau, aux Rairies (Maine-et-Loire). Histogrammes des inclinaisons avant correction (a) et des inclinaisons après corrections (b); diagramme de pointage (c).

provenir de l'action du champ démagnétisant car l'aimantation mesurée sur les carreaux est trop faible $(2,6 \mathrm{~A} / \mathrm{m})$. Par contre, elle s'explique par la présence d'une très forte anisotropie magnétique caractérisée par les facteurs moyens $\overline{\mathrm{F}}_{\mathrm{x} z}=1,35$ et $\overline{\mathrm{F}}_{\mathrm{xy}}=1,08$. En conséquence, une correction d'anisotropie a été effectuée sur l'ensemble des mesures, de même qu'une correction de pente de $8^{\circ}$ pour tenir compte du positionnement des carreaux dans le four à sol oblique (tig. 9) (Lanos, 1987a). On obtient finalement l'histogramme des inclinaisons (fig. 11b) et le diagramme de pointage (fig. 11c). On a ainsi :

\begin{tabular}{|l|c|c|c|c|}
\cline { 2 - 5 } \multicolumn{1}{c|}{} & \multicolumn{2}{c|}{ À plat } & \multicolumn{2}{c|}{ Sur tranche } \\
\cline { 2 - 5 } \multicolumn{1}{c}{} & $\begin{array}{c}\text { Incli- } \\
\text { naison }\end{array}$ & $\begin{array}{c}\text { Dévia- } \\
\text { tion }\end{array}$ & $\begin{array}{c}\text { Incli- } \\
\text { naison }\end{array}$ & $\begin{array}{c}\text { Dévia- } \\
\text { tion }\end{array}$ \\
\hline Mesures initiales .................. & $48^{\circ} 1$ & $15^{\circ} 8$ & $56^{\circ} 6$ & $12^{\circ} 8$ \\
Correction d'anisotropie ... & $54^{\circ} 8$ & $15^{\circ} 3$ & $54^{\circ} 5$ & $13^{\circ} 3$ \\
Correction de pente ...... & $62^{\circ} 4$ & $19^{\circ} 2$ & $62^{\circ} 3$ & $16^{\circ} 6$ \\
\hline
\end{tabular}


L'effet d'anisotropie joue essentiellement sur la direction d'aimantation des carreaux à plat. L'écart avant et après correction est dans ce cas de $7^{\circ}$. En revanche, l'effet joue peu sur les carreaux cuits sur tranche puisque l'écart est de $2^{\circ}$ seulement. Cela vient de ce que le plan d'aplatissement des carreaux est très proche $\left(16^{\circ} 6 \mathrm{E}\right)$ de la direction du NM de l'époque et de ce que l'anisotropie dans le plan d'aplatissement est faible $(1,08)$ (cf. fig. 8). Après une correction de latitude de $1^{\circ} 2$ pour normalisation à Paris, l'inclinaison moyenne devient:

$$
\overline{\mathrm{I}}=63^{\circ} 6 \pm 0^{\circ} 6
$$

Cette valeur, déterminée pour le mois de janvier 1986, correspond remarquablement bien, au demidegré près, à celle de $64^{\circ}$ observée à Paris pour la même époque. Cette expérience constitue une excellente vérification de la véracité des déductions archéologiques tirées des mesures archéomagnétiques, tant en ce qui concerne l'orientation de l'axe du four par rapport au NM régnant lors de la cuisson, qu'en ce qui concerne le mode de rangement, des matériaux. De plus, les différentes corrections montrent qu'il est possible de retrouver très précisément la direction du CMT ayant régné lors de la cuisson des matériaux.

\section{EXEMPLES \\ DE DATATIONS ARCHEOMAGNETIQUES}

\section{Senlis (Oise)}

Cette étude porte sur un lot de 99 fragments de tegulae provenant d'une fouille de sauvetage effectuée en 1986 dans l'ancien palais épiscopal de Senlis (Oise). Il s'agit d'une toiture effondrée en place (couche 23) sur le sol d'un bâtiment ayant donné de nombreux indices d'une occupation durant le III $\mathrm{s}$. Ces structures ont été coupées par le rempart galloromain édifié à la fin du $\mathrm{II}^{\mathrm{e}} \mathrm{s}$. Les mesures archéomagnétiques ont conduit aux résultats exposés cidessous (Goulpeau, Lanos, 1986).

La statistique des positions de cuisson montre que $91 \%$ des tuiles ont été cuites debout. Le pointage des directions d'aimantation (fig. 12a) fait apparaître deux groupements principaux de points pour des valeurs complémentaires de la déviation. Les tuiles étudiées ont donc été cuites majoritairement debout dans un four de forme rectangulaire orienté grossièrement nord-sud, est-ouest. Toutes les tuiles de l'agglomérat $B$, composé uniquement de triangles pleins, présentent leurs rebords tournés vers le NM. Par contre, l'agglomérat A est formé d'un mélange de triangles pleins et vides, c'est-à-dire qu'une partie des tuiles avaient leurs rebords tournés vers l'est et l'autre partie vers l'ouest. Signalons l'existence d'un petit groupe de points constitué de tuiles cuites de chant, en $\bar{I}=57^{\circ}$ et $\bar{\Delta}=60^{\circ}$. Il peut s'agir soit d'un réemploi ou d'une réfection, soit d'une cuisson contemporaine du lot principal, selon des positions anormales pour compléter le chargement. Le faible nombre de ces éléments ne permet pas de trancher entre ces deux solutions.

Le diagramme des densités de points (fig. 12b) permet de bien visualiser le centre de gravité des deux groupements principaux. On obtient les deux directions moyennes suivantes :

$$
\begin{array}{ll}
\text { agglomérat } A: \bar{I}=63^{\circ} & \bar{\Delta}=+10^{\circ} \\
\text { agglomérat } \mathrm{B}: \overline{\mathrm{I}}=71^{\circ} & \bar{\Delta}=-74^{\circ}
\end{array}
$$

L'importante différence d'inclinaison entre les agglomérals $\mathrm{A}$ et $\mathrm{B}$ ne peut résulter uniquement d'un effet d'anisotropie (l'effet de forme ne joue pas ici du fait de la faible intensité d'aimantation des matériaux). Cette différence doit plutôt provenir d'un pendage systématique des matériaux dans le but d'assurer une meilleure stabilité du chargement. Comme pour les effets de forme et d'anisotropie, le pendage influe directement sur la valeur de l'inclinaison des matériaux cuits debout perpendiculairement au NM, mais n'affecte pratiquement pas la valeur de l'inclinaison des matériaux cuits parallèlement à ce NM (cf. fig. 8). L'inclinaison moyenne de $63^{\circ}$ fournie par l'agglomérat A esl donc vierge de toute perturbation et peut servir à la datation. Après correction de latitude entre Senlis et Paris, la valeur de l'inclinaison ramenée à Paris est égale à $62^{\circ} 5$. Cette valeur, reportée sur la courbe de variation séculaire de l'inclinaison (fig. 3), donne une seule solution compatible avec les données archéologiques, soit $200 \pm 20$ ans après J.-C.

\section{Plénée-Jugon (Côtes-d'Armor)}

Il s'agit d'un lot de 116 tegulae recueillies en prospection sur un site gallo-romain situé au lieu-dit La Mare-Pilais. La statistique des positions de cuisson indique que $95 \%$ des tuiles ont été cuites de chant. Le diagramme de pointage (fig. 13a) montre nettement l'existence de deux agglomérats très concentrés, de déviations moyennes quasi complémentaires. Cela correspond de toute évidence à une cuisson des matériaux dans un four de forme rectangulaire, orienté à environ $60^{\circ} \mathrm{W}$ et $30^{\circ} \mathrm{E}$ par rapport au NM de l'époque. Les données archéomagnétiques peuvent ainsi renseigner indirectement sur l'orientation des fours, sans que ces derniers aient été préalablement retrouvés et fouillés sur le terrain. Les 


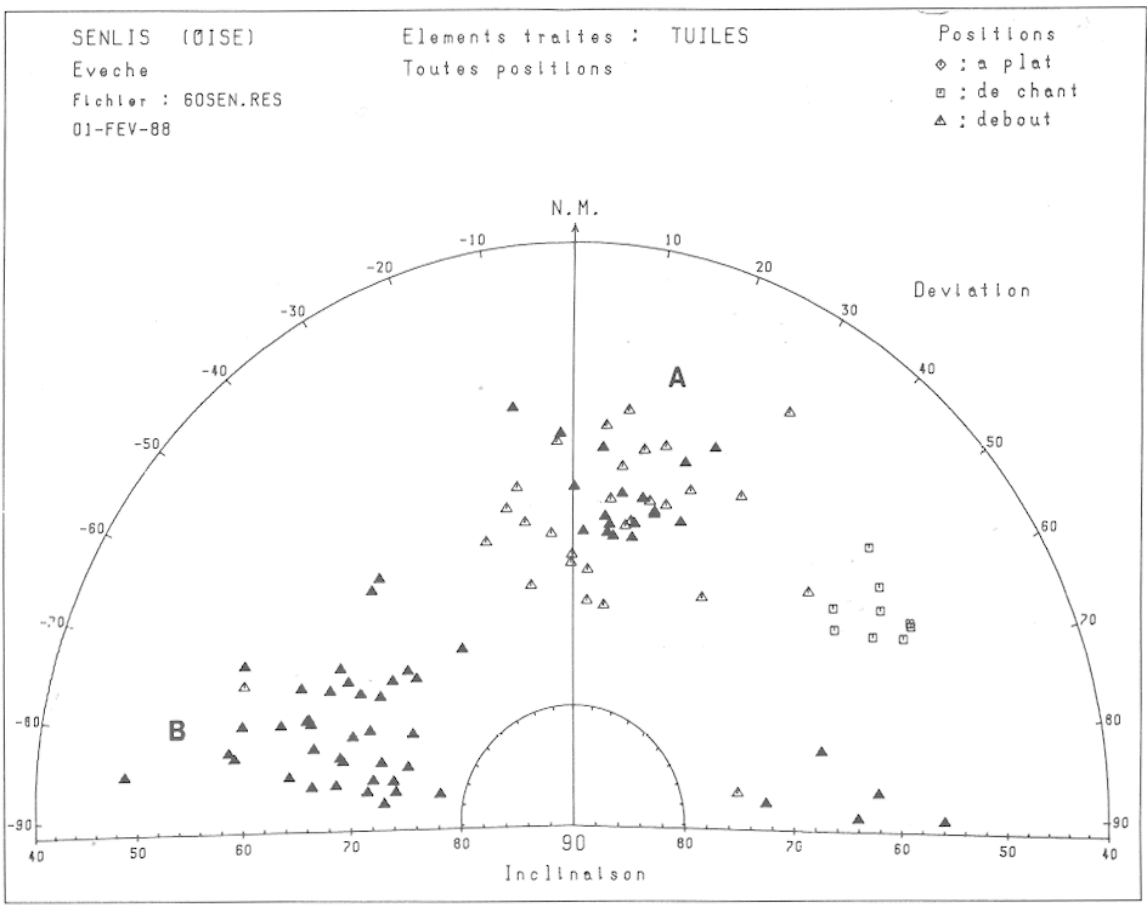

Fig. 12 - Senlis (Oise) Diagrammes de pointage (a) et des densités de points (b).

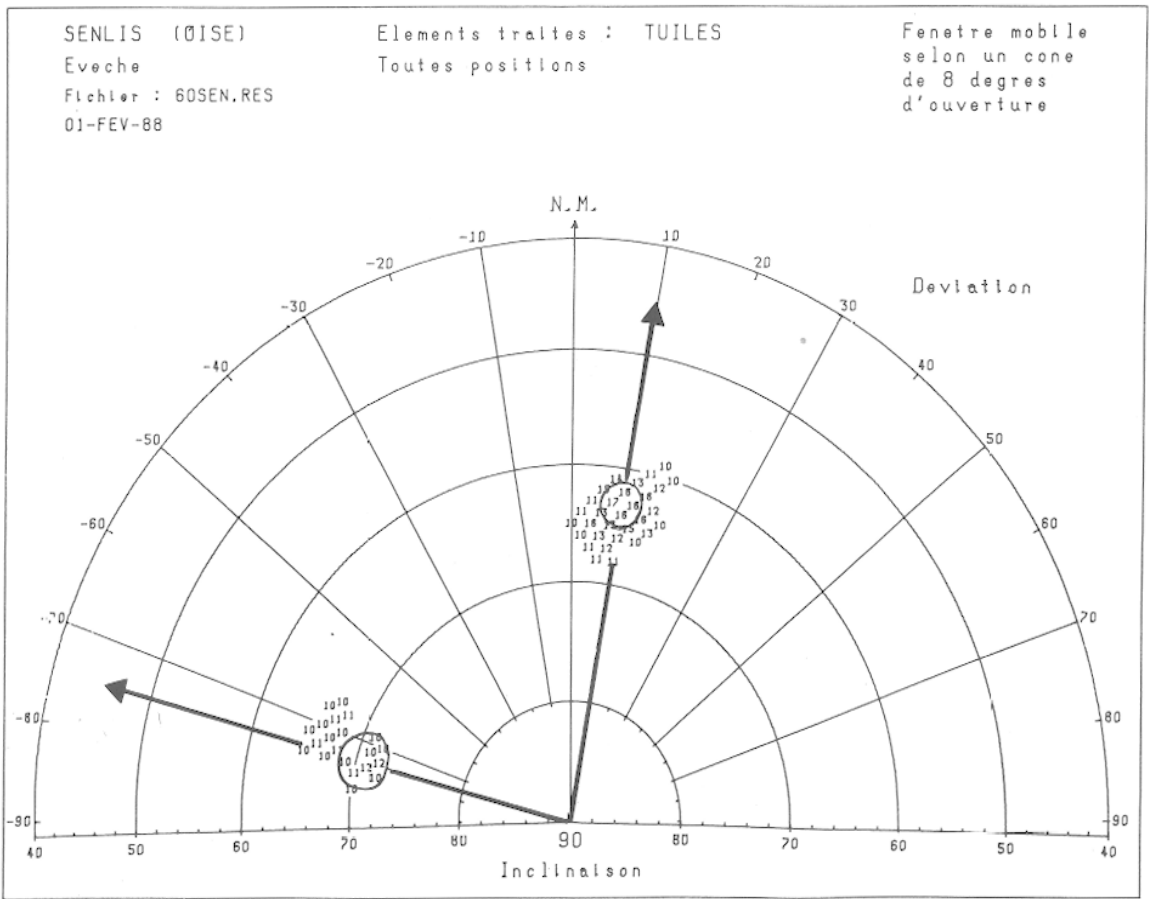

tuiles de l'agglomérat proche de $+30^{\circ}$ ont leurs rebords presque tous tournés vers l'Est magnétique (carrés vides) tandis que celles de l'agglomérat complémentaire ont leurs rebords orientés moitié vers le Nord (carrés pleins), moitié vers le Sud magnétique (carrés vides). Le diagramme des densités de points (fig. 13b) fournit les directions moyennes suivantes :

agglomérat $\mathrm{A}: \overline{\mathrm{I}}=67^{\circ} \quad \bar{\Delta}=+28^{\circ}$

agglomérat $\mathrm{B}: \mathrm{I}=70^{\circ} \quad \Delta=-62^{\circ}$
Une fois encore, on constate que l'agglomérat B présente une inclinaison moyenne supérieure à celle de l'agglomérat $\mathrm{A}$. Cet écart est très probablement imputable à l'anisotropie (l'intensité d'aimantation des tuiles n'est pas assez forte ici pour produire un effet de forme aussi important). Bien que la déviation moyenne de l'agglomérat $A$ ne soit pas très proche de $0^{\circ}$, les calculs montrent que la valeur moyenne de $67^{\circ}$ n'est pratiquement pas affectée par les effets d'anisotropie et de pendage. Après une 
Fig. 13 - Plénée-Jugon (Côtes-d'Armor).

Diagrammes de pointage (a) et des densités de points (b)

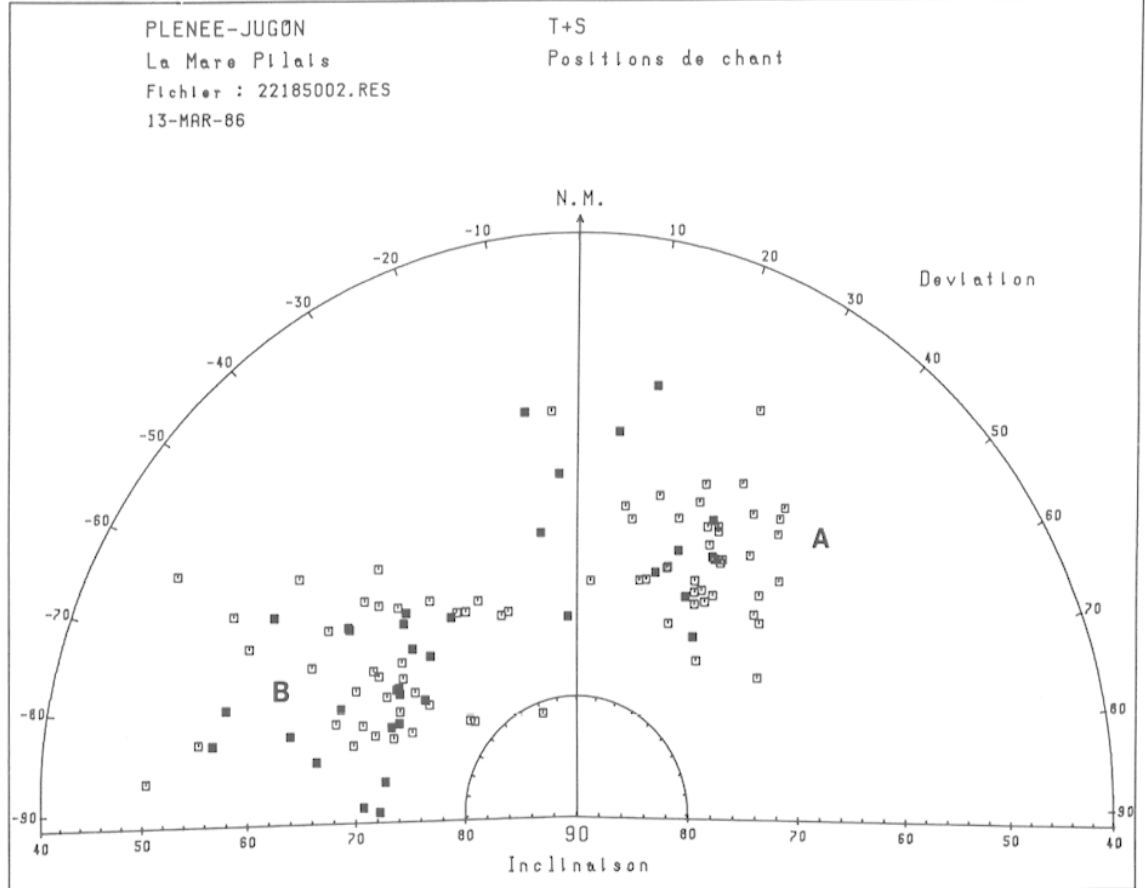

a

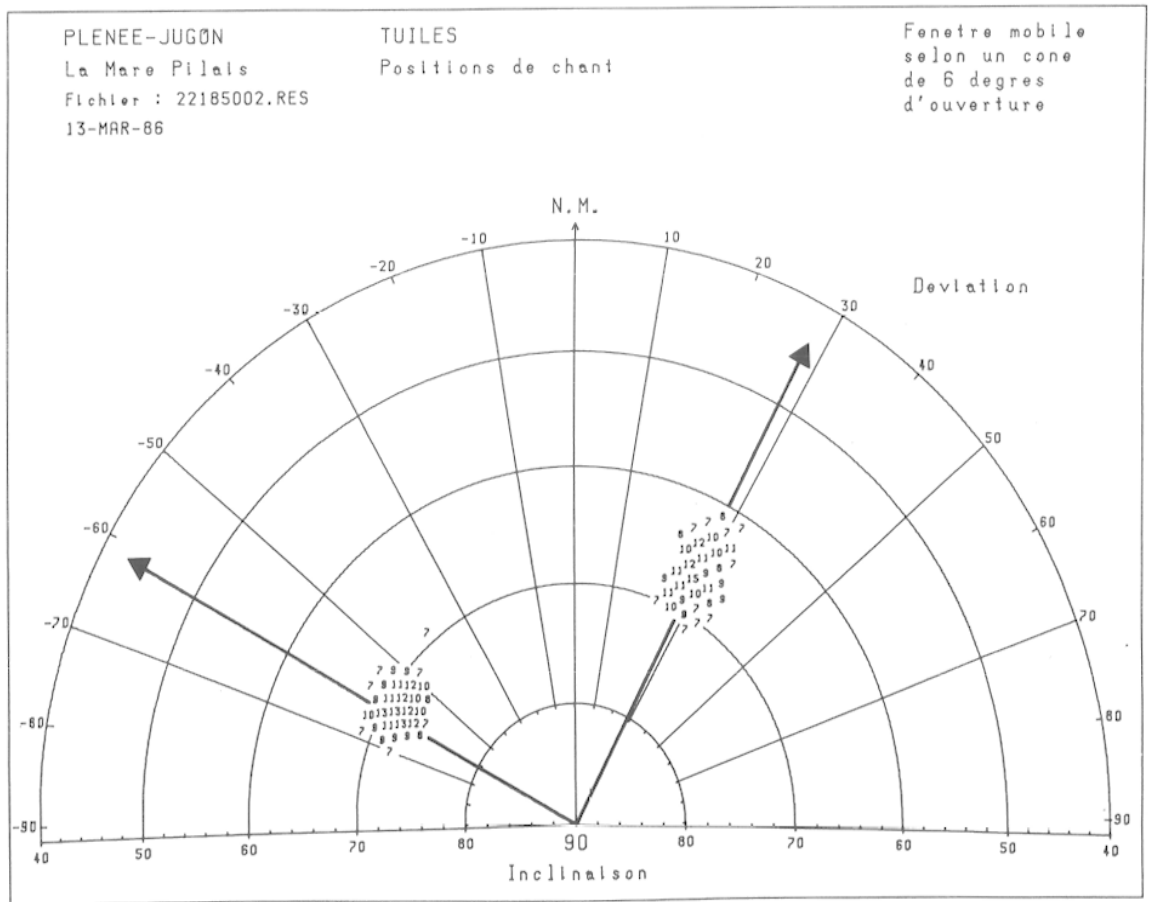

correction de latitude de $0^{\circ} 5$, on peut retenir la valeur moyenne de $67^{\circ} 5$ qui, reportée sur la courbe de la figure 3 , fournit les deux dates de fabrication suivantes : $0 \pm 20$ ans après J.-C., ou $240 \pm 20$ ans après J.-C. L'absence de mobilier archéologique associé ne permet pas pour l'instant de choisir entre ces deux dates.

Les positions réelles des matériaux sur tranche pouvaient différer plus ou moins largement des positions théoriques. Pour asseoir les chargements, il était parfois plus commode d'incliner les matériaux les uns contre les autres selon des directions pouvant varier d'une rangée à l'autre et d'une couche à l'autre. Lorsque cette dernière alternance existe, elle peut être décelée dans les diagrammes inclinaisondéviation par l'apparition d'agglomérats dédoublés. 


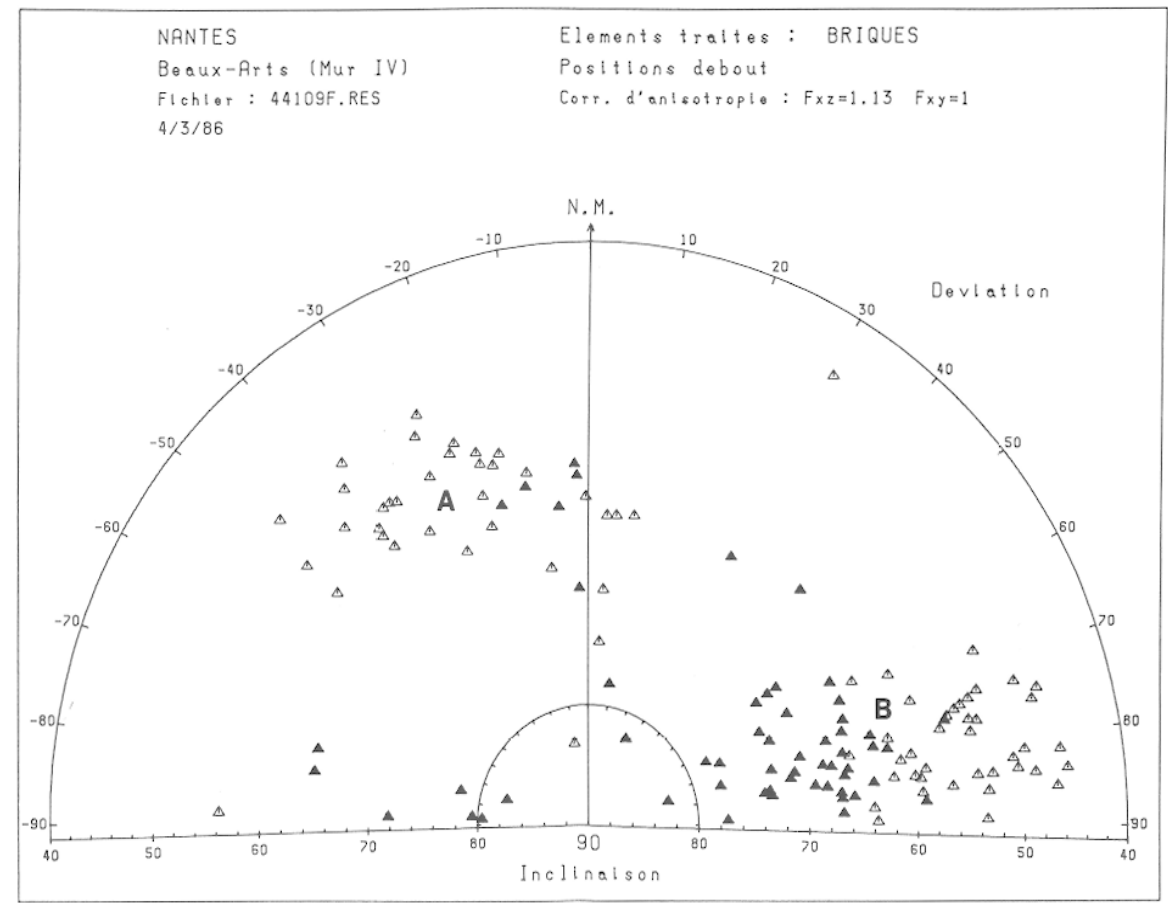

Fig. 14 - Nantes (Loire-Atlantique). Diagramme de pointage.

\section{Nantes (Loire-Atlantique)}

Cette étude d'un lot homogène de 145 briques gallo-romaines (mur IV des fouilles du jardin des Beaux-Arts à Nantes) montre les conséquences d'un pendage des matériaux sur les mesures archéomagnétiques. Après correction d'une légère anisotropie d'ATR $\left(\overline{\mathrm{F}}_{\mathrm{x} z}=1,13 ; \overline{\mathrm{F}}_{\mathrm{xy}}=1\right)$, le diagramme de pointage (fig. 14) montre que les briques ont été cuites debout (à $91 \%$ d'après les statistiques), en majorité perpendiculairement au NM de l'époque. L'ensemble $B$ est nettement dédoublé selon deux agglomérats mis en évidence par les triangles pleins et vides. L'interprétation du dédoublement est immédiate. Il s'agit de briques ayant été cuites selon un pendage alterné d'une rangée à l'autre. On observe pour l'agglomérat $B$ une très nette corrélation entre l'orientation des faces lissées des briques et le sens du pendage. En effet, les faibles inclinaisons correspondent à un pendage vers le nord et à une orientation des faces lissées des briques vers le sud (triangles vides). Inversement, les fortes inclinaisons correspondent à un pendage vers le sud et à une orientation des faces lissées vers le nord (triangles pleins). En supposant un pendage symétrique du type de celui de la figure 15 , la correction nécessaire à apporter pour regrouper au mieux les directions d'aimantation est de $\pm 8^{\circ}$.

L'inclinaison moyenne de l'aimantation obtenue après correction pour les agglomérats $\mathrm{A}$ et $\mathrm{B}$ se situe entre $60^{\circ}$ et $61^{\circ}$. Après correction de latitude, on

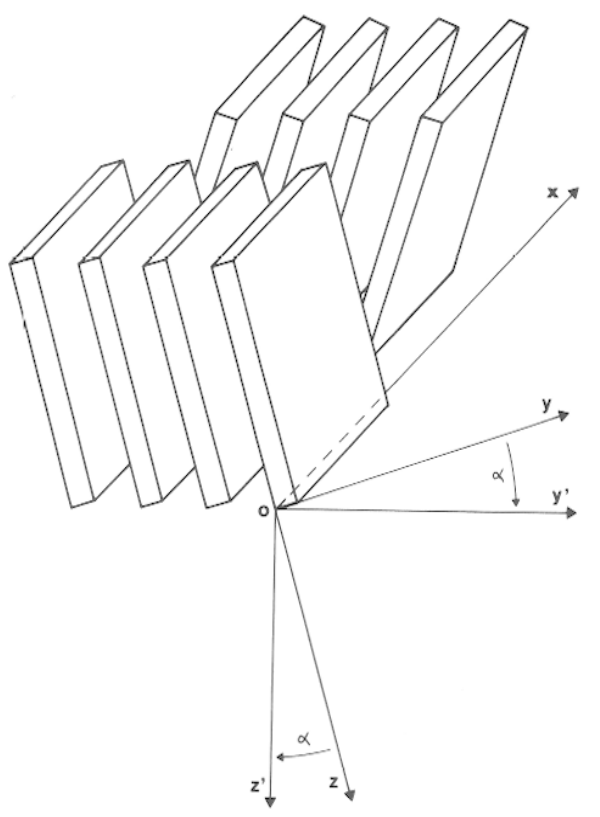

Fig. 15 - Pendage alterné de rangées de matériaux cuits debout.

obtient une valeur de l'inclinaison à Paris entre $61^{\circ} 5$ et $62^{\circ} 5$. Cette valeur reportée sur la courbe de variation séculaire (fig. 3) donne comme seule solution cohérente avec les données chronologiques (articulation $\mathrm{I}^{\mathrm{er}}-\mathrm{II}^{\mathrm{e}} \mathrm{S}$.) la date de $110 \pm 20$ ans après J.-C. 
Fig. 16 - Toulouse, amphithéâtre de Purpan

(Haute-Garonne)

Histogrammes

des inclinaisons (a) et des intensités d'aimantation (b); diagramme de pointage (c).

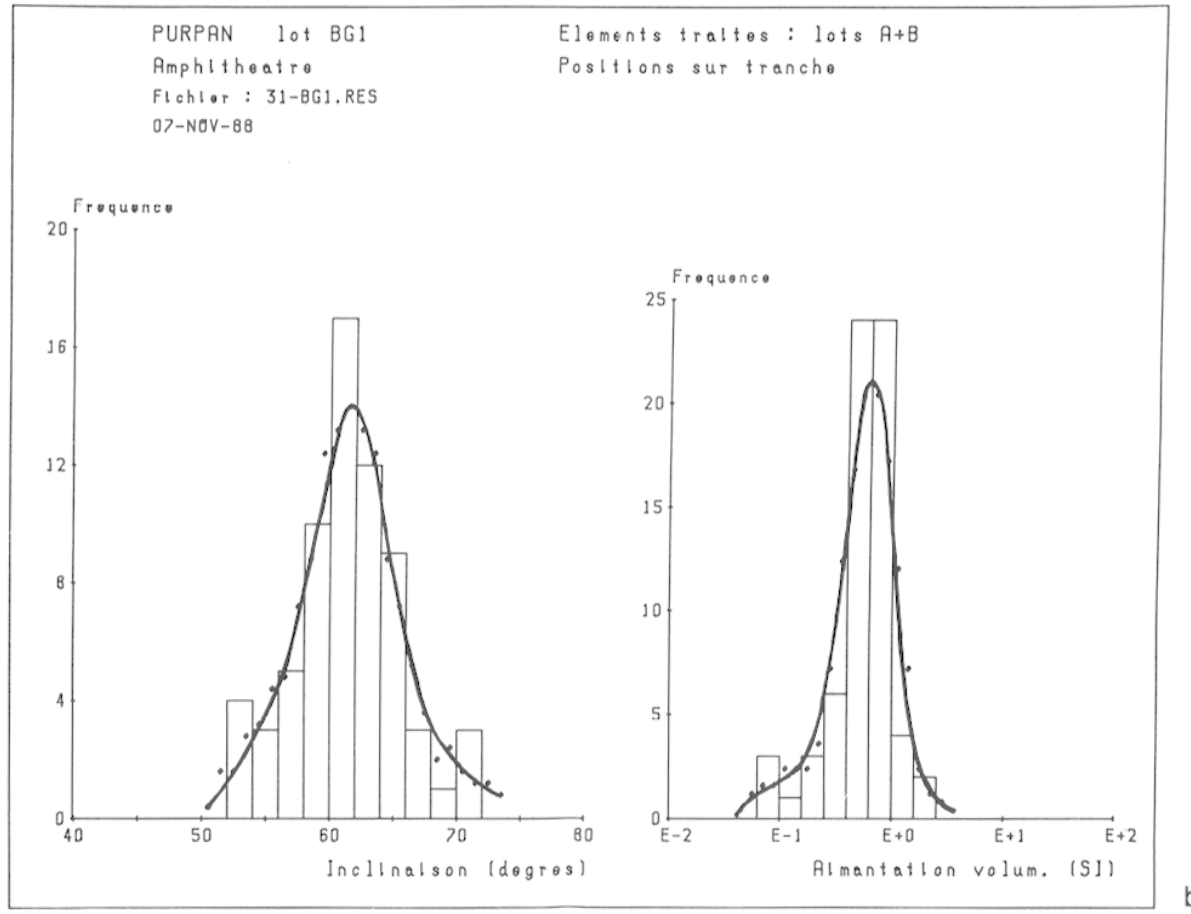

a

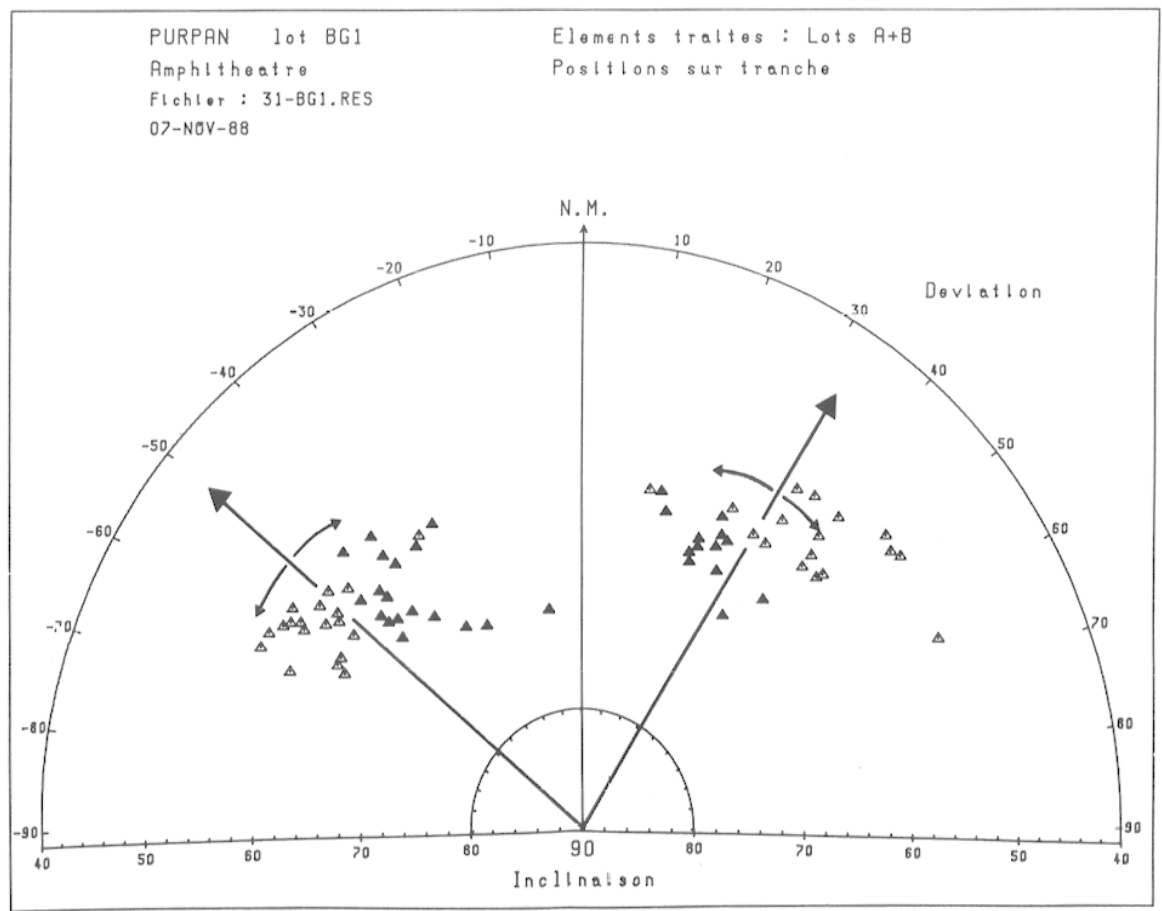

\section{Purpan (Haute-Garonne)}

Cet exemple concerne un lot de 63 briques galloromaines estampillées $T A$ et $A T$, prélevées dans un tronçon de mur effondré de l'amphithéâtre proche de Toulouse. Toutes les briques ont été cuites en position debout. L'histogramme très resserré des intensités d'aimantation (fig. 16b) montre la très grande homogénéité magnétique du lot étudié.
L'histogramme des inclinaisons (fig. 16a) donne une excellente estimation de l'inclinaison moyenne, soit $\overline{\mathrm{I}}=61^{\circ} 5$.

Le diagramme de pointage (fig. 16c) révèle l'existence de deux groupements principaux dédoublés autour des déviations moyennes $33^{\circ} \mathrm{E}$ et $50^{\circ} \mathrm{W}$. Chacun des groupements est en fait dédoublé selon que la face lissée de la brique était tournée vers le 
NM (triangle plein) ou vers le Sud (triangle vide). Cette disposition peut s'expliquer soit par un pendage alterné des matériaux (faces lissées vers le ciel comme dans l'exemple précédent), soit par une organisation des rangées de briques en chevrons alternés. Il est évident que nous avons affaire à une logique de chargement du four extrêmement régulière, caractéristique des pratiques artisanales de l'atelier de production.

Après une correction de latitude de $5^{\circ}$, la valeur moyenne de l'inclinaison ramenée à Paris devient $66^{\circ} 5$, d'où les solutions chronologiques suivantes: $30 \pm 20$ après J.-C.; $220 \pm 20$ après J.-C. ; $270 \pm 20$ après $\mathrm{J}$.-C. Seules les données de la fouille permettront éventuellement de choisir l'une de ces trois solutions.

\section{Haute-Bretagne}

A côté d'études effectuées sur des lots fournis par les archéologues dans le cadre de fouilles programmées, le Laboratoire d'Archéométrie a trouvé un vaste champ d'application de la méthode dans le cadre d'une Action thématique programmée (CNRS) et d'un Projet collectif de recherche (Ministère de la Culture) sur l'archéologie du milieu rural à l'époque gallo-romaine en Haute-Bretagne. Cette zone test recouvre trois départements, à savoir l'Illeet-Vilaine et la partie des Côtes-d'Armor et du Morbihan située à l'est d'une ligne Saint-Brieuc/Vannes. La connaissance du milieu rural à l'époque gallo-romaine dans cette région a fait de nombreux progrès grâce aux renseignements recueillis sur plus de 1500 sites de prospection. Ces sites fournissent un matériel varié constitué majoritairement de mobilier céramique et de matériaux de construction. En particulier, la collecte, en grand nombre, de fragments de tuiles à des fins de datation est l'œuvre de nombreux prospecteurs bénévoles travaillant soit individuellement (bassin de la Vilaine et Morbihan), soit au sein d'associations (Centre régional d'archéologie d'Alet, Centre de recherche archéologique du Pays de Rennes). Cette phase de prélèvement sur le terrain ne demande qu'un minimum de précaution : localisation précise de la collecte et constitution éventuelle de lots différenciés au sein d'un même site.

Dans l'état actuel des recherches (Goulpeau, Langouët, 1984; Goulpeau, Langouët, Lanos, 1984; Lanos, Langouët, Goulpeau, 1989), une cinquantaine de lots provenant de toute la Haute-Bretagne ont fourni des résultats archéomagnétiques exploitables. Les valeurs ramenées à Paris des inclinaisons moyennes déterminées sur les lots s'échelonnent entre $60^{\circ}$ et $70^{\circ}$. Il convient de noter en particulier que plus de $65 \%$ de ces valeurs sont supérieures à $64^{\circ}$, ce qui implique dans ce cas deux solutions chronologiques pour la fabrication des matériaux : l'une durant la première moitié $d u \mathrm{I}^{\mathrm{er}} \mathrm{s}$. après $\mathrm{J}$.-C. et l'autre durant le III $^{\mathrm{e}} \mathrm{s}$. (cf. fig. 3). A l'instar des exemples traités précédemment, le choix d'une solution chronologique particulière parmi les dates archéomagnétiques possibles n'est envisageable que s'il existe des arguments archéologiques solides pour l'étayer. En l'occurrence, ce choix a été rendu possible pour 37 lots grâce à l'analyse statistique des données chronologiques fournies par les tessons de céramique sigillée retrouvés sur les sites en association avec les tuiles et les briques (Langouët, Goulpeau, 1975; Langouët, Galliou, Gallou, 1984). Les deux exemples suivants indiquent le mode de raisonnement suivi.

Le premier exemple concerne le site d'habitat gallo-romain de la Touche, à Créhen (Côtesd'Armor). Les dates de fabrication de 125 tessons de sigillée recueillis lors de la fouille de ce site sont données (fig. 17a). L'étude archéomagnétique de tuiles et tubuli retrouvés sur place (inclinaison moyenne de $62^{\circ}$ après correction de latitude) donne trois dates possibles pour la construction de l'édifice (fig. 17b). Vu l'apport de sigillées sur le site en proportions importantes dès le début du ${ }^{\text {er }} \mathrm{s}$., il paraît normal de retenir la solution de $110 \pm 20$ ans après J.-C. pour la fabrication des tuiles. Il est particulièrement intéressant de remarquer qu'il existe un écart supérieur au demi-siècle entre le début de la fabrication de ces sigillées importées et la date de construction. Cet écart est très probablement dû en grande partie au temps de diffusion de la céramique entre le moment où elle est fabriquée dans les ateliers du sud et du centre de la Gaule et le moment où elle arrive effectivement sur le site d'utilisation. Il ne faut cependant pas négliger le fait que des habitats de type traditionnel pouvait fort bien s'approvisionner en sigillées bien avant toute mutation architecturale.

Le deuxième exemple concerne le site d'habitat gallo-romain du Tertre-de-Crémur à Hillion (Côtesd'Armor). 495 tessons de sigillée ont été retrouvés lors de prospections au sol (fig. 18a). Parallèlement, une datation archéomagnétique réalisée sur un lot de 60 tuiles à rebord (inclinaison moyenne de $68^{\circ}$ ramenée à Paris) indique une fabrication de ces éléments soit au tout début de notre ère, soit au milieu du $\mathrm{III}^{\mathrm{e}} \mathrm{s}$. après J.-C. (fig. 18b). La quasiabsence de sigillées au $\mathrm{I}^{\text {er }} \mathrm{s}$. nous amène à rejeter la première solution. Il reste donc la seule solution du milieu du III' $^{\mathrm{s}}$. pour la construction de l'édifice. 


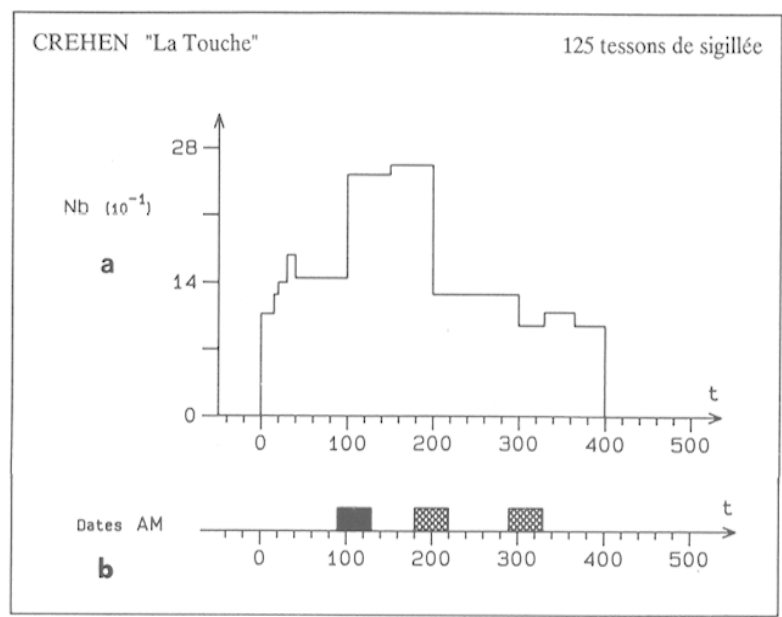

Fig. 17 - Importations de céramiques sigillées sur le site de la Touche à Créhen (Côtes-d'Armor) et datation archéomagnétique (AM).

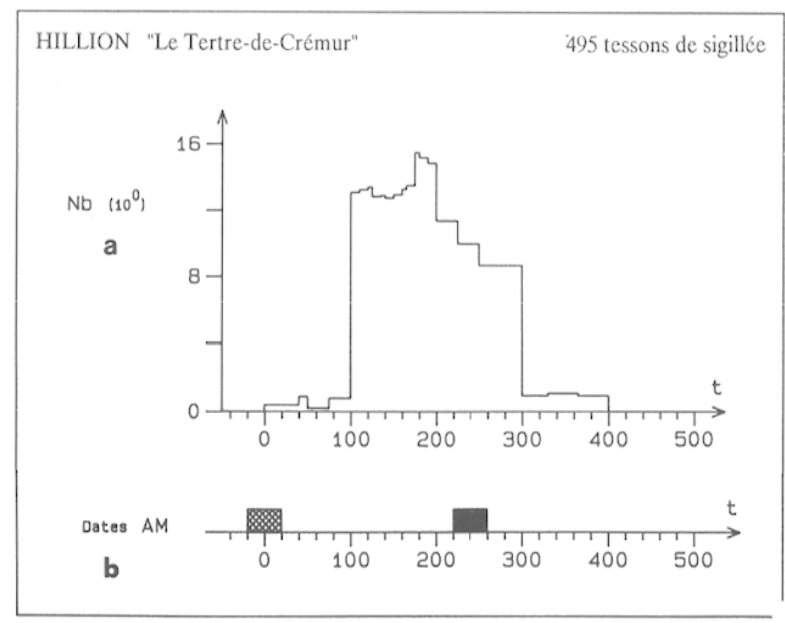

Fig. 18 - Importations de céramiques sigillées sur le site du Tertre-de-Crémur à Hillion (Côtes-d'Armor) et datation archéomagnétique (AM).

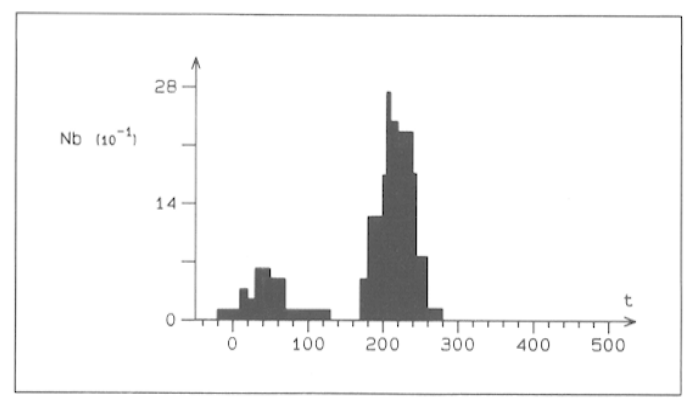

Fig. 19 - Histogramme des fréquences annuelles de fabrication des tuiles et briques retrouvees sur 37 sites ruraux gallo-romains de Haute-Bretagne.
Comme précédemment, on remarque qu'il existe un décalage entre le moment où les céramiques du site ont commencé à être fabriquées et le moment où la construction de l'édifice a eu lieu.

Les résultats des datations ainsi obtenues pour les 37 lots sont rassemblés sous la forme d'un histogramme (fig. 19). Il ressort de son examen que $22 \%$ des ensembles ont été fabriqués entre 20 avant J.-C. et 130 après $\mathrm{J}$. -C. et que $78 \%$ des ensembles ont été fabriqués entre 170 et 280 après $\mathrm{J}$.-C. Ainsi, une première vague de construction au $\mathrm{I}^{\mathrm{ir}} \mathrm{s}$. après J.-C. est suivie d'une deuxième très importante à la fin du $I^{r}$-début du III $^{r} \mathrm{~s}$. après J.-C. Les lacunes observées au milieu du Ir $^{r}$ s. el à partir de la fin du $\mathrm{III}^{\mathrm{e}} \mathrm{s}$. ne doivent pas faire penser à une absence totale de construction durant ces périodes. Un échantillonnage de 37 lots pris au hasard des prospections parmi le très grand nombre des édifices ayant réellement existé ne peut que servir à révéler les grandes tendances de l'histoire architecturale. d'une région. Compte tenu de ces remarques, il ressort de cette statistique que la plupart des constructions employant les tuiles et les briques ne seraient apparues dans le milieu rural de HauteBretagne que vers la fin du II" et le début du III"s. après J.-C. En conséquence, on est conduit à admettre, pour nos régions, une persistance de l'habitat traditionnel jusqu'au milieu du II" s. après J.-C. Après une première romanisation et urbanisation au cours du $\mathrm{I}^{\mathrm{er}} \mathrm{s}$., la population autochtone aurait mis près d'un siècle avant d'intégrer des éléments matériels aussi tangibles de la civilisation romaine. A titre de comparaison, l'apparition des constructions en maçonnerie en Ile-de-France est datée de la fin du Ire $s$. et du début du II" s. après J.-C. (Le Glay, 1975). Ce retard à la romanisation mis en évidence par les études archéomagnétiques dans le domaine de l'architecture, traduit une remarquable persistance $d u$ fonds indigène. A l'appui de cette constatation, il convient de noter que de telles persistances matérielles et culturelles ont été observées même en des régions très tôt fortement colonisées comme la Gaule méridionale (Barruol, 1976).

\section{LES APPORTS TECHNOLOGIQUES}

Si la datation est l'objectif principal de l'étude archéomagnétique des matériaux d'argile cuite, il n'en demeure pas moins que cette méthode permet l'obtention de renseignements archéologiques précieux portant essentiellement sur les modes de 
chargements des fours et les caractéristiques magnétiques des pâtes. La reconstitution des modes de chargement des fours peut se faire à partir du pourcentage des positions de cuisson (à plat, de chant, debout), de l'orientation des plans d'aplatissement et de l'orientation des faces des objets. La caractérisation des pâtes peut se faire en fonction de l'intensité moyenne d'aimantation et de l'éventuelle présence de l'anisotropie d'aimantation thermorémanente.

\section{LES POSITIONS DE CUISSON}

Bien que toutes les positions soient a priori envisageables pour la cuisson des matériaux de construction, il apparaît dans la pratique que les positions réellement utilisées vérifiaient un certain nombre de conditions d'équilibre et de logique des chargements. En particulier, la disposition des matériaux ne doit pas gêner le bon tirage des fours. Lorsque l'objet est de forme parallélépipédique, on peut distinguer les positions à plat, de chant et debout. Par contre, on ne distingue que les positions à plat et sur tranche pour les objets de forme carrée tels que les carreaux de pavage et certaines briques (de pilettes d'hypocauste par exemple).

Pour l'époque gallo-romaine, en France, pratiquement tous les lots de tuiles et de briques étudiés sont cuits dans une proportion $90 \%$ debout, $10 \%$ à plat et de chant. Dans l'ètat actuel des données, tout lot présentant une autre position majoritaire de cuisson peut apparaître comme l'œuvre d'un atelier aux pratiques originales. Ainsi, parmi la centaine de sites gallo-romains étudiés jusqu'ici, un seul présente des éléments majoritairement cuits en position de chant (cf.' ci-dessus l'exemple de Plénée-Jugon).

Pour l'époque médiévale, les positions de cuisson sont beaucoup plus variées. Les tuiles plates à crochet sont très souvent cuites de chant, comme le montre un reste de chargement retrouvé récemment dans un des fours de la Cour Napoléon au Louvre à Paris (Espagnet, 1987). Notons cependant l'existence de lots combinant les deux positions de rangement de chant et debout à la fois. La combinaison $1 / 3$ de chant, $2 / 3$ debout a été mise en évidence pour des lots de tuiles plates provenant d'abbayes médiévales de la région de Dijon (Côte-d'Or). Cependant, nous ne savons pas encore quel pouvait être l'agencement de ces types de chargement. Les positions de cuisson des carreaux de pavage sont, quant à elles, assez variées. On observe soit des chargements uniquement sur tranche (cf. La Bintinais à Rennes, in : Lanos, 1987a) ou uniquement à plat, soit une combinaison de couches alternées à plat - sur tranche comme aux Rairies (cf. supra, p. 327, Vérification expérimentale).

\section{Le PENDAge des MatériauX \\ LA PENTE DU SOL DES FOURS}

L'existence de pendages est attestée aussi bien pour les tuiles (Goulpeau, Langouët, 1980) et les briques gallo-romaines que pour les tuiles plates et les carreaux de pavages médiévaux. Les effets du pendage sont décelables lorsque les données archéomagnétiques présentent des dédoublements d'agglomérats facilement identifiables et interprétables, comme c'est le cas pour les exemples de Nantes et Purpan ci-dessus. Ils sont aussi décelables lorsqu'il existe un écart important entre les inclinaisons moyennes des agglomérats de déviation proche de $90^{\circ}$ et ceux de déviation proche de $0^{\circ}$ (cf. supra, p. 329, l'exemple de Senlis). Dans ce cas, l'absence de dédoublement signifie que les matériaux penchaient tous dans le même sens pour une direction de rangement donnée. De façon analogue à l'effet de forme et à l'effet de l'anisotropie d'ATR, l'effet du pendage est minimal, voire nul, lorsque le plan d'aplatissement des objets (sur tranche) est quasi parallèle à la direction du NM de l'époque.

Des problèmes de pente interviennent lorsque les matériaux sont cuits dans des fours à tirage oblique. Le chargement repose alors directement sur un sol oblique dont la pente est dirigée vers l'alandier. Dans les diagrammes archéomagnétiques, ce type de chargement entraîne généralement l'apparition d'un seul agglomérat de points pour les matériaux cuits sur tranche. En effet, la position sur tranche exige, pour des raisons d'équilibre, que les matériaux soient tous placés parallèlement à la direction de pente (Goulpeau, Langouët, Lanos, 1986). La position à plat reste à l'inverse une position toujours stable. Le remplissage du four des Rairies est exemplaire à ce titre (fig. 9). La correction de l'effet de pente est souvent très difficile, voire impossible, puisqu'il faut connaître à l'avance l'angle de pente du sol du four à l'origine de la production. Heureusement, cette perturbation semble assez rare puisqu'elle n'a été véritablement rencontrée qu'une seule fois au Laboratoire à propos de briques postmédiévales provenant de l'abbaye de Fontevrault. 
LES DIRECTIONS DE RANGEMENT

LE PLAN DES FOURS

Les déviations observées dans les diagrammes de pointage donnent l'orientation des matériaux dans les fours. Pour les positions de chant ou debout, la déviation représente l'angle entre le plan vertical d'aplatissement des objets et le Nord magnétique. Pour la position à plat, la déviation représente l'angle entre l'un des bords des objets et le Nord magnétique. La présence d'agglomérats très concentrés en inclinaison et déviation induit une disposition des matériaux selon des rangées rectilignes (fig. 7). Par contre, une répartition uniforme des déviations entre $-90^{\circ}$ et $+90^{\circ}$ induit l'existence de rangées disposées en cercles.

Le bon sens nous entraîne à penser que la disposition de ces rangées était guidée par la forme interne des parois des laboratoires. A l'époque galloromaine, la forme des fours était le plus souvent rectangulaire (Mc Whirr, 1979; Le Ny, 1988). La forme trapézoïdale existe aussi, mais elle semble rare. C'est pourquoi la forme rectangulaire est a priori déduite de l'existence d'agglomérats complémentaires. Pour l'époque médiévale, les diagrammes archéomagnétiques suggèrent une grande variété de modes de chargement. Un cas particulier intéressant est le rangement en chevrons alternés dans un four rectangulaire. Un exemple archéologique tout récent découvert lors des fouilles de la Cour Napoléon au Louvre est venu apporter une preuve de l'existence de tels rangements (Espagnet, 1987).

\section{L'ORIENTATION DES FACES DES MATÉRIAUX}

La face dite supérieure des matériaux est définie comme étant la face comportant des traces de lissage, côté rebord pour les tuiles gallo-romaines, côté crochet éventuel pour les tuiles plates médiévales, côté décor éventuel pour les carreaux de pavage. Notons que cette dénomination conventionnelle "face supérieure" est sans rapport avec l'orientation vers le haut ou vers le bas des objets dans les constructions. On peut repérer, au sein d'un même agglomérat, les différentes orientations des faces des matériaux. Les éléments d'un agglomérat peuvent avoir toutes les faces supérieures tournées dans une même direction ou bien présenter, par exemple, une moitié dans un sens et une moitié dans l'autre. On a vu que cette alternance permet parfois de déceler des effets parasites tels que les pendages (cf. supra, p. 332, les exemples de Nantes et Purpan). Plus généralement, cette donnée peut servir à caractériser des ateliers en fonction des types de chargement (Lanos, Langouët, Goulpeau, 1989).

\section{LES INTENSITÉS MOYENNES D'AIMANTATION}

Lorsque l'histogramme des aimantations volumiques (exprimées en $\mathrm{A} / \mathrm{m}$ ) présente un seul mode très pointu et resserré, on peut définir pour le lot une intensité moyenne d'aimantation caractéristique. Pour l'époque gallo-romaine, en France, les valeurs d'intensité observées sur une trentaine de lots (les sites de Haute-Bretagne étant mis à part), se répartissent entre 0,4 et $20 \mathrm{~A} / \mathrm{m}$. Mais on observe une différenciation très intéressante en fonction de la nature géologique des régions. En effet, les intensités se répartissent entre 0,4 et $2 \mathrm{~A} / \mathrm{m}$ pour les bassins sédimentaires et entre 1,5 et $20 \mathrm{~A} / \mathrm{m}$ pour les massifs cristallins. Ce résultat, qu'il faut bien sûr considérer d'un point de vue statistique, s'explique par la plus ou moins grande concentration en minéraux magnétiques de ces différentes formations géologiques.

Pour l'époque médiévale, toujours en France, des observations effectuées sur 32 lots montrent des intensités d'aimantation en moyenne beaucoup plus faibles. En effet, les valeurs se répartissent entre 0,5 à $9 \mathrm{~A} / \mathrm{m}$, avec près de $70 \%$ d'entre elles comprises entre 1 et $5 \mathrm{~A} / \mathrm{m}$. L'origine de ces différences entre les deux époques romaine et médiévale n'a pas encore été étudiée de façon approfondie. On sait seulement que les modes de cuisson (température, atmosphère) de même que le choix des argiles employées, interviennent de façon complexe dans le processus d'acquisition de l'aimantation.

D'autre part, il arrive parfois qu'on puisse mettre en évidence, au sein d'un même lot, des souspopulations d'éléments ayant des aimantations moyennes différentes. C'est le cas pour un lot de 240 fragments de carreaux médiévaux recueillis par L. Goulpeau et L. Langouët dans la tessonnière de l'atelier $\mathrm{n}^{\circ} 3$ de la Hardelière à Laval (Mayenne) (Bucur et al., 1984). Ces auteurs ont pu montrer l'existence d'une nette corrélation entre la coloration des pâtes et l'amplitude de l'aimantation.

\section{L'ANISOTROPIE D'AIMANTATION THERMORÉMANENTE}

D'une manière générale, on attribue l'origine de l'anisotropie d'ATR des matériaux de construction à un alignement préférentiel des grains magnétiques provoqué par le mode de moulage de la pâte argileuse. Le tableau suivant donne les facteurs 
d'anisotropie moyens $\overline{\mathrm{F}}_{\mathrm{xz}}$ et $\overline{\mathrm{F}}_{\mathrm{xy}}$ déterminés sur différents lots de matériaux de différentes époques :

\begin{tabular}{|c|c|c|c|}
\hline & $\overline{\mathrm{F}}_{\mathrm{xz}}$ & $\overline{\mathrm{F}}_{\mathrm{xy}}$ & \\
\hline \multirow{9}{*}{$\begin{array}{l}\text { Tuiles et briques } \\
\text { gallo-romaines }\end{array}$} & 1,07 & 1,03 & Guignen, le Biffoué (35) \\
\hline & 1,09 & 1,00 & $\begin{array}{l}\text { Pléchâtel, } \\
\text { La Ville-Chérel (35) }\end{array}$ \\
\hline & 1,13 & 1,00 & Nantes, Beaux-Arts (44) \\
\hline & 1,17 & 1,00 & Le Mans, école Chappe (72) \\
\hline & 1,18 & 1,00 & Châtillon-sur-Seiche (35) \\
\hline & 1,19 & 1,04 & $\begin{array}{l}\text { Metz, Saint-Pierre-aux- } \\
\text { Nonnains (57) }\end{array}$ \\
\hline & 1,26 & 1,04 & Le Chayla (48) \\
\hline & 1,35 & 1,03 & $\begin{array}{l}\text { Baguer-Pican, } \\
\text { La Moignerais (35) }\end{array}$ \\
\hline & 1,70 & 1,03 & $\begin{array}{l}\text { Saint-Julien-de-Peyrolas } \\
\quad(30)\end{array}$ \\
\hline \multirow[t]{8}{*}{$\begin{array}{l}\text { Carreaux médiévaux } \\
\text { et post-médiévaux }\end{array}$} & 1,10 & 1,00 & $\begin{array}{l}\text { Brains/Allones, la cave } \\
\text { peinte (49) }\end{array}$ \\
\hline & 1,18 & 1,01 & $\begin{array}{l}\text { Rennes, La Bintinais, } \\
\text { lot G (35) }\end{array}$ \\
\hline & 1,17 & 1,01 & $\begin{array}{l}\text { Rennes, La Bintinais, } \\
\text { lot E (35) }\end{array}$ \\
\hline & 1,19 & 0,99 & $\begin{array}{c}\text { Fontevrault, Prieuré } \\
\text { Saint-Lazare (49) }\end{array}$ \\
\hline & 1,25 & 1,01 & $\begin{array}{l}\text { Durtal, La Motte- } \\
\text { Grouillon (49) }\end{array}$ \\
\hline & 1,29 & 1,00 & $\begin{array}{l}\text { Rennes, La Bintinais, } \\
\text { lot F (35) }\end{array}$ \\
\hline & 1,29 & 1,05 & $\begin{array}{l}\text { Avrillé, La Haye-aux- } \\
\text { Bonhommes (49) }\end{array}$ \\
\hline & 1,29 & 1,02 & $\begin{array}{l}\text { Olivet, abbaye de } \\
\text { Clermont (53) }\end{array}$ \\
\hline Tuiles médiévales & 1,27 & 1,15 & $\begin{array}{l}\text { Broualan, Le Bois-de- } \\
\text { Buzot (35) }\end{array}$ \\
\hline Carreaux modernes & 1,35 & 1,08 & $\begin{array}{l}\text { Les Rairies, briqueterie } \\
\text { Cailleau (49) }\end{array}$ \\
\hline
\end{tabular}

Les tuiles plates médiévales et les carreaux modernes montrent des facteurs moyens systématiquement supérieurs à 1 . Ainsi, la direction de plus facile aimantation se situe selon l'axe $\mathrm{x}$, parallèlement aux stries de lissage visibles en surface, tandis que la direction de moins facile aimantation se situe selon l'axe $z$, perpendiculairement au plan d'aplatissement (Oxy) des matériaux (fig. 1). Ces directions principales d'anisotropie, qui traduisent des orientations privilégiées des grains magnétiques dans l'argile, coïncident parfaitement avec un étirement de la pâte observé selon l'axe $\mathrm{x}$ et avec un feuilletage observé selon le plan d'aplatissement. Dans le cas des Rairies, l'étirement et le feuilletage résultent à l'évidence de l'extrusion mécanique de l'argile à travers une fente. Quant aux tuiles plates médiévales, leur très faible épaisseur et leur allongement important (dimensions moyennes: $25 \times 15 \times$ $1,3 \mathrm{~cm}$ ) ont dû favoriser un couchage manuel fortement directionnel de la pâte lors du moulage.
Les autres lots présentent des facteurs $\overline{\mathrm{F}}_{\mathrm{xz}}$ toujours supérieurs à 1 et des facteurs $\overline{\mathrm{F}}_{\mathrm{xy}}$ très voisins de 1 . Les valeurs de $\tilde{F}_{x y}$ s'expliquent par l'absence d'une direction privilégiée d'étirement de la pâte dans le plan (Oxy). L'épaisseur plus grande et le rapport longueur/largeur voisin de 1 des objets ont sans doute empêché un étalement unidirectionnel de l'argile dans ce plan. Par contre, les valeurs de $\bar{F}_{x z}$ révèlent un important feuilletage selon le plan d'aplatissement. Il n'existe apparemment pas de corrélation entre ce dernier facteur et l'époque de fabrication des matériaux. Rien que pour l'époque gallo-romaine, on observe la plus grande diversité des valeurs moyennes d'un lot à un autre. En fait, l'anisotropie d'ATR dépend non seulement de la technique de moulage, mais aussi de la nature des minéraux magnétiques mis en jeu. Aussi les recherches menées pour évaluer la part respective de ces deux causes d'anisotropie devraient-elles permettre d'apporter à l'avenir de nouveaux et précieux renseignements tant archéologiques qu'archéomagnétiques.

\section{Discussion sUR LES APPORTS TECHNOLOGIQUES}

Les paragraphes précédents ont tenté de montrer les retombées archéologiques des études archéomagnétiques. La rigueur exige d'évaluer toutes les solutions technologiques possibles déductibles des données fournies par l'archéomagnétisme. Ces apports sont à replacer parmi les autres sources de renseignements archéologiques que sont la prospection, la fouille et l'analyse du matériel. Ainsi, l'étude des marques digitées et des estampilles relevées sur les tuiles et les briques est un autre moyen d'approcher les problèmes de production et de diffusion. L'amélioration des connaissances sur les matériaux de construction ne peut provenir que de la synthèse de toutes ces recherches. L'archéomagnétisme des matériaux déplacés fournit des indications pouvant aider largement à la reconstitution de l'histoire d'un atelier ou encore de l'itinéraire d'une production. Parallèlement, l'utilisation statistique d'un grand nombre de mesures archéomagnétiques est à même de fournir des données archéologiques fiables concernant des phases de construction, des aires de production ou encore des pratiques artisanales.

\section{$\stackrel{*}{* *}$}

Cette présentation détaillée des différents aspects de la datation archéomagnétique des matériaux déplacés montre qu'il est possible, lorsquc la variation séculaire du CMT est bien connue, de dater 
avec une précision de \pm 20 ans des lots homogènes de tuiles, de briques ou de carreaux de pavage. Cependant, il n'est pas rare d'obtenir plusieurs solutions chronologiques pour la datation d'un même lot. Cela résulte de ce que l'inclinaison du CMT a pu reprendre plusieurs fois la même valeur au cours de la période historique. Bien que le contexte archéologique permette le plus souvent de choisir une solution particulière parmi celles proposées, il n'en reste pas moins que ce choix se révèle parfois très délicat, voire impossible. L'utilisation conjointe des deux composantes Inclinaison et Intensité du CMT devrait permettre de s'affranchir du contexte et de sélectionner de façon non ambiguë la date de fabrication des matériaux. C'est pourquoi le Laboratoire d'Archéométrie s'est fixé pour tâche de mettre au point une méthode de routine spécialement appliquée aux matériaux déplacés permettant d'exploiter avec précision et fiabilité la variation séculaire de l'intensité du CMT.

Les nombreux exemples cités montrent les multiples apports chronologiques et technologiques de la mèthode. C'est une des originalités de l'archéomagnétisme que de proposer, en plus d'une datation précise, une série de renseignements d'ordre technologique concernant les modes de fabrication des matériaux. Jusqu'à ce jour, plus de 200 lots de matériaux gallo-romains ou médiévaux provenant de toute la France ont fait l'objet d'une étude archéomagnétique au Laboratoire d'Archéométrie de Rennes. L'ampleur de l'échantillonnage ainsi constitué permet déjà de voir des phénomènes historiques intéressants à grande échelle : périodes de construction des fana dans l'ouest de la Gaule par exemple, ou encore pratiques artisanales particulières durant, le Moyen Age pour la cuisson des tuiles plates. Ainsi, au-delà de l'étude particulière d'un site, qui possède naturellement sa problématique archéologique propre, l'archéomagnétisme offre la possibilité d'appréhender des faits historiques à grande échelle grâce à des études statistiques sur de grands ensembles chronologiques ou géographiques.

\section{LES AIMANTATIONS THERMORÉMANENTES PARTIELLES}

Supposons un échantillon d'argile cuite ayant déjà acquis une ATR dans un champ terrestre $\mathrm{CMT}_{1}$. S'il est chauffé à une température de $300^{\circ} \mathrm{C}$ par exemple, puis refroidi dans un nouveau champ $\mathrm{CMT}_{2}$ différent du premier, alors une partie des grains magnétiques prendra une nouvelle aimantation dirigée selon $\mathrm{CMT}_{2}$. L'autre partie des grains ne sera pas affectée par ce traitement et conservera la mémoire du champ $\mathrm{CMT}_{1}$. Ainsi, l'aimantation thermorémanente totale mesurée sur l'échantillon sera la somme de deux aimantations partielles $\mathrm{ATRP}_{1}$ et $\mathrm{ATRP}_{2}$ acquises chacune dans deux gammes de température différentes. Pour retrouver la direction de chacune de ces ATRP, il faudra effectuer au laboratoire une procédure de désaimantation thermique qui consiste en une série de chauffes successives à des températures croissantes suivies de refroidissements en champ nul.
Par définition, on appelle aimantation primaire l'ATR acquise après chauffe complète de l'argile audelà de la température de Curie $T_{\text {. }}$. Cette aimantation correspond le plus souvent à la cuisson des matériaux lors de la fabrication. Les aimantations secondaires désignent les ATRP acquises lors des différents réchauffements $\left(0^{\circ}<\mathrm{T}<\mathrm{T}_{c}\right)$ postérieurs successifs des matériaux. Ces aimantations témoignent d'incendies, de feux de défrichage ou encore d'exposition à la chaleur dans des structures telles que les thermes, les parois de fours ou les foyers domestiques. Les positions des matériaux lors de ces réchauffements, la cohérence ou non des directions d'aimantation correspondantes, de même que les observations archéologiques concernant le lot étudié, permettent généralement de reconstituer et éventuellement de dater les circonstances de ces réaimantations partielles. 


\section{Applications archéologiques}

des désaimantations thermiques

Les aimantations secondaires sont considérées comme parasites si elles sont archéomagnétiquement inexploitables. C'est le cas la plupart du temps lorsque l'on a affaire à des destructions de toitures par incendie - cf. l'exemple du fanum de Chênehutte-les-Tuffeaux (Maine-et-Loire) in : Goulpeau, Lanos, Langouët, 1986 - ou à des brûlis de surface lors de défrichages. Par contre, ces aimantations deviennent fort intéressantes lorsqu'elles sont acquises dans des positions cohérentes.

Prenons l'exemple de carreaux de pavage et de briques en place respectivement sur le sol et dans les murs d'un bâtiment incendié. Dans ce cas, l'inclinaison moyenne des aimantations secondaires permet de remonter à la date de l'incendie tandis que la déviation moyenne redonne l'orientation $d u$ bâtiment par rapport au Nord magnétique de l'époque. Si l'incendie correspond à l'abandon de l'édifice, alors la durée maximale d'occupation de ce dernier est donnée par l'intervalle de date compris entre la fabrication des matériaux et cet incendie cf. l'exemple de l'abbaye de Notre-Dame-de-laCapelle (I.es Attaques, Pas-de-Calais) in : Goulpeau, Lanos, Langouët, 1986 - . Une ètude semblable a été réalisée sur des briques provenant des parois d'un four de tuilier gallo-romain situé au Biffoué à Guignen (Ille-et-Vilaine) (Goulpeau et alii, 1982). Cette étude a permis d'estimer à environ 60 ans la durée d'utilisation du four entre le moment où les matériaux des parois ont été fabriqués et le moment où l'installation a été chauffée pour la dernière fois.

La validité des durées d'utilisation déduites de ces différents exemples suppose implicitement qu'il n'y a pas eu de période d'abandon ou de stockage entre le moment de la fabrication et le moment de la dernière utilisation. Dans le cas contraire, on peut envisager toutes sortes de scénarios complexes. On citera pour exemple les résultats d'une désaimantation thermique effectuée récemment par M. E. Evans et M. Mareschal (1986) sur des fragments de tuiles prélevées sur le sol d'un foyer domestique d'Italie du sud, daté du $\mathrm{vI}^{\mathrm{e}} \mathrm{s}$. après J.-C. Ces auteurs ont pu montrer l'existence de trois ATRP attribuées respectivement à la cuisson en position debout dans un four lors de la fabrication (ATRP entre $500^{\circ} \mathrm{C}$ et $\mathrm{T}_{\mathrm{c}}$ ), à ce qui doit probablement être un incendie (directions d'ATRP quelconques entre $300^{\circ}$ et $500^{\circ} \mathrm{C}$ ) et à la réutilisation dans le foyer domestique (entre $20^{\circ}$ et $300{ }^{\circ} \mathrm{C}$ ).

Outre les nombreux apports à la datation, la désaimantation thermique permet aussi de reconstituer les positions normales d'utilisation pour des éléments provenant de structures effondrées ou détruites au cours de leur refroidissement - cf. l'étude archéomagnétique de fours à sel de La Tène finale mis au jour à Karreg-ar-Yellan à Ploubazlanec (Côtes-d'Armor) in : Lanos, 1987a - Enfin, la désaimantation thermique offre la possibilité d'étudier la distribution des températures maximales atteintes au sein d'un four ou d'une installation de chauffage. Une étude de ce type est actuellement en cours sur des briques de pilett.es d'hypocauste provenant d'un caldarium récemment fouillé à Entrains (Nièvre).

Philippe Lanos*

* Laboratoire d'Archéométrie, Université de Rennes I.

\section{BIBLIOGRAPHIE}

Aitken M. J.

1974 : Physics and Archaeology, Londres, Clarendon Press, 291 p.

Barruol G.

1976 : Assimilation et résistance à la culture gréco-romaine dans le monde ancien, Travaux du $V^{*}$ Congrès Internalional d'Etudes Classiques, Madrid, Septembre 1974, p. 389-405.
Bauer L. A.

1899 : On the Secular Variation of a Free Magnetic Needle, Physical Review, 3, p. 34-38.

Bucur I.

1986 : Fourteenth Century Archaeomagnetic Field Directions from Widely Distributed Sites in France, in: J. S. OLin, M. J. Blackman (éd.) Proceedings of the 24th International 
Archaeometry Symposium, Washington, Smithsonian Institution Press, p. 449-458.

Bucur I., Dufournier D., Goulpeau L., Langouët L., Naveau J. 1984 : La céramique à "œil de perdrix" et la production de la Hardelière à Laval (Mayenne), Archéologie Médiévale, XIV, p. 169-219.

Espagnet $\mathrm{F}$.

1987 : Archéologie : Palissy aux Tuileries de Paris, La Revue de la Céramique et du Verre, 32, p. 12-14.

Evans M. E., Mareschal M.

1986 : An Archaeomagnetic Example of Polyphase Magnetization, Geomagnetism and Geoelectricity, 38, p. 923-929.

Goulpeau L., Langouët L.

1980 : Datations d'apports de tuiles et briques sur des sites gallo-romains à l'aide de mesures archéomagnétiques opérées sur d'abondants échantillonnages, Revue d'Archéométrie, 4, p. 153-164.

1982 : Problems of Interpretation of Different Graphs (I, $\Delta$ ) Obtained through an Archaeomagnetic Study of Displaced Materials, in : A. Aspinal., S. E. Warren (éd.), Proceedings of the 22nd Symposium on Archaeometry, Bradford, U.K., p. 112-121.

1984 : Détermination des dates de construction des édifices gallo-romains par l'archéomagnétisme des matériaux en argile (tuiles et briques), in: La prospection archéologique en HauteBretagne, Dossiers du Centre Régional Archéologique d'Alet, no spécial G, p. 128-133.

Goulpeau L., Lanos P.

1986: Dalation archéomagnétique de fragments de tuiles trouvées en fouille (couche 23) dans l'ancien palais des évêques de Senlis (60), rapport interne d'expertise, inédit.

Goulpeau L., Langouët L., Lanos P.

1984 : Datation archéomagnétique des établissements galloromains à partir des matériaux de construction d'argile, Bulletin de la Société Scientifique de Bretagne, 56, p. 107-116.

1986 : Apports de l'archéomagnétisme à l'étude de l'évolution des technologies de fabrication des matériaux de construction en argile cuite, in: D. Drrosux (éd.), Terres cuites architecturales au Moyen Age, Actes du Colloque de Saint-Omer, 7-9 juin 1985, Arras, p. 165-173.

Goulpeau L., Lanos P., Langouët L.

1986 : Les apports de la désaimantation thermique dans des études archéomagnétiques de matériaux déplacés, Revue $d$ ' $A r$ chéométrie, 10, p. 9-18.

1989 : The Anisotropy as a Disturbance of the Archaeomagnetic Dating Method, in: Y. Maniatis (éd.), Archaeometry, Proceedings of the 25th Internalional Symposium, Amsterdam, Elsevier, p. 45-58.

Goulpeau L., Langouët L., Bardel J.-P., Bucur I.

1982 : Possibilités de l'archéomagnétisme dans l'étude d'un four de tuilier, Revue d'Archéométrie, 6, p. 13-25.

Hoye G. S.

1981 : Archaeomagnetic Secular Variation Record of Mount Vesuvius, Nature, 291, p. 216-218.

Langouët L., Goulpeau L.

1975 : Sur l'utilisation statistique et cartographique des séries d'objets archéologiques, Dossiers du Centre Régional Archéologique d'Alet, no 3 , p. 95-118.
Langouët L., Bucur I., Goulpeau L.

1983 : Les problèmes de l'allure de la courbe de variation séculaire du champ magnétique terrestre en France, nouveaux résultats archéomagnétiques, Revue d'A rchéométrie, 7, p. 37-43.

\section{Langouët L., Galliou P., Gallou M.}

1984: Analyse statistique d'un mobilier caractéristique: la céramique sigillée, in: La prospection archéologique en IlauleBretagne, Dossiers du Cenlre Régional Archéologique d'Alet. no spécial G, p. 90-99.

\section{Lanos P.}

1987a : Archéomagnétisme des matériaux déplacés, applicalions à la datation des matériaux de construction d'argile cuite en archéologie, Thèse de Doctorat, Univ. Rennes I, 317 p.

1987b : The effects of Demagnetizing Fields on Thermoremanent Magnetization Acquired by Parallel Sided Baked Clay Blocks, Geophysical Journal of the Royal Astronomical Sociely, 91, p. 985-1012.

Lanos P. Langouët L., Goulpeau L.

1989 : Datation archéomagnétique des édifices ruraux galloromains en Ilaute-Bretagne, in: Meithodes pluridisciplinaires d'étude scientifique des sites el vestiges archéologiques, 112 Congrès National des Sociétés Savantes, Lyon, 21-25 avril 1987, Paris, Éd. du Comité des Travaux Ilistoriques et Scientifiques, p. 85-106.

Lanos P., Goulpeau L., Bucur I., Langouët L.

à paraitre : $\Lambda$ Double Wave Variation for the Geomagnetic Field Inclination during the Roman Period, Geophysical Journal International.

\section{Le Glay M.}

1975 : La Gaule romanisée, in: IIistoire de la France rurale, Paris, Éd. Seuil, p. 190-285.

Le Ny F.

1988 : Les fours de tuiliers gallo-romains, Paris, Ed. Maison des Sciences de l'Homme, Documents d'Archéologie Française, 12, $144 \mathrm{p}$.

Mc Whirr A.

1979 : Roman Tile-Kilns in Britain, BAR International Series, 68 , p. $97-189$.

Néel L.

1949 : Théorie du trainage magnétique des ferromagnétiques en grains fins avec application aux terres cuites, Annales de Géophysique, 5, p. 99-136.

Tanguy J.-C., Bucur I., Thompson J. F. C.

1985: Geomagnetic Secular Variation in Sicily and Revised Ages of Historic Lavas from Mount Etna, Nature, 318, p. 453455 .

Thellier E.

1938: Sur l'aimantation des terres cuites el ses applications géophysiques, Thèse de Doctorat, Paris; Annales de l'Instilut de Physique du Globe de Paris, 16, p. 157-302.

1971 : Magnétisme interne, in : Géophysique, Encyclopédie de la Pléiade, p. 235-376.

1981 : Sur la direction du champ magnétique terrestre en France durant les deux derniers millénaires, Physics of the Earth an Planelary Inleriors, 24, p. 89-132. 